\title{
Netrin/DCC Signaling Guides Olfactory Sensory Axons to Their Correct Location in the Olfactory Bulb
}

\author{
Vanisha Lakhina, ${ }^{1}$ Christina L. Marcaccio, ${ }^{1}$ Xin Shao, ${ }^{1}$ Mark E. Lush, ${ }^{1,3}$ Roshan A. Jain, ${ }^{2}$ Esther Fujimoto, ${ }^{4,5}{ }^{\dagger}$ \\ Joshua L. Bonkowsky, ${ }^{4,5}$ Michael Granato, ${ }^{2}$ and Jonathan A. Raper ${ }^{1}$ \\ Departments of ${ }^{1}$ Neuroscience and ${ }^{2}$ Cell and Developmental Biology, University of Pennsylvania School of Medicine, Philadelphia, Pennsylvania 19104, \\ ${ }^{3}$ Stowers Institute for Medical Research, Kansas City, Missouri 64110, and ${ }^{4}$ Department of Neurobiology and Anatomy, and ${ }^{5}$ Department of Pediatrics, \\ Division of Pediatric Neurology, University of Utah School of Medicine, Salt Lake City, Utah 84132
}

Olfactory sensory neurons expressing particular olfactory receptors project to specific reproducible locations within the bulb. The axonal guidance cues that organize this precise projection pattern are only beginning to be identified. To aid in their identification and characterization, we generated a transgenic zebrafish line, OR111-7:IRES:Gal4, in which a small subset of olfactory sensory neurons is labeled. Most sensory neurons expressing the OR111-7 transgene project to a specific location within the bulb, the central zone protoglomerulus, while a smaller number project to the lateral glomerulus 1 protoglomerulus. Inhibiting Netrin/DCC (deleted in colorectal cancer) signaling perturbs the ability of OR111-7-expressing axons to enter the olfactory bulb and alters their patterns of termination within the bulb. The Netrin receptor DCC is expressed in olfactory sensory neurons around the time that they elaborate their axons, netrin $1 \mathrm{a}$ is expressed near the medial-most margin of the olfactory bulb, and netrin $1 \mathrm{~b}$ is expressed within the ventral region of the bulb. Loss of Netrin/DCC signaling components causes some OR111-7-expressing sensory axons to wander posteriorly after exiting the olfactory pit, away from netrin-expressing areas in the bulb. 0R111-7-expressing axons that enter the bulb target the central zone less precisely than normal, spreading away from netrin-expressing regions. These pathfinding errors can be corrected by the reexpression of DCC within OR111-7 transgene-expressing neurons in DCC morphant embryos. These findings implicate Netrins as the only known attractants for olfactory sensory neurons, first drawing 0R111-7-expressing axons into the bulb and then into the ventromedially positioned central zone protoglomerulus.

\section{Introduction}

Olfactory sensory neurons in the olfactory epithelia of vertebrates stochastically choose to express a single specific receptor from a large repertoire of odorant receptors (ORs), thereby conferring a distinct molecular and functional identity upon each neuron. They project axons to the olfactory bulb where they synapse onto second order neurons. The axons of sensory neurons expressing the same OR converge into glomeruli that are located in reproducible locations within the olfactory bulb. This pattern of con-

Received Aug. 29, 2011; revised Jan. 25, 2012; accepted Feb. 1, 2012.

Author contributions: V.L. and J.A.R. designed research; V.L., C.L.M., X.S., and M.E.L. performed research; R.A.J., E.F., J.L.B., and M.G. contributed unpublished reagents/analytic tools; V.L., C.L.M., and X.S. analyzed data; V.L. and J.A.R. wrote the paper.

'Deceased, August 21, 2011.

This work was funded by NIH Grants R01-DA025407 awarded to J.A.R., 5F32NS065637 to R.A.J., and R01HD37975 to M.G. We dedicate this paper to our colleague and friend Chi-Bin Chien. We thank Minghong Ma and Steven Scherer for helpful suggestions. Thanks to Yoshihiro Yoshihara for the OMP:lyn-RFP- and TRPC2:Gap-Venustransgenic zebrafish lines and the pzOMP2.1-Venus plasmid. We also thank the Raper laboratory for fruitful discussions and Alison Dell for making the schematics in Figure 1. Thanks to Amy H. Lim and Chi-Bin Chien for the pME-dcc clone and Chi-Bin Chien for netrin1a, netrin1b, and DCC in situ plasmids. The UAS:DCC rescue transgenic line was generated in the Chien laboratory and was supported by NIH Grants K12 5HD001410 and K08 DA024753, and a Primary Children's Medical Center Foundation Grant to J.L.B.

The authors declare no competing financial interests.

Correspondence should be addressed to Jonathan A. Raper, 1123 BRB2/3, 421 Curie Blvd., University of Pennsylvania School of Medicine, Philadelphia, PA 19104. E-mail: raperj@mail.med.upenn.edu.

DOI:10.1523/JNEUROSCI.4442-11.2012

Copyright $\odot 2012$ the authors $\quad 0270-6474 / 12 / 324440-17 \$ 15.00 / 0$ vergence into OR-specific glomeruli is essential for normal olfactory perception (Sakano, 2010). Understanding how sensory axons find their way to their appropriate glomeruli is a key step in understanding how the olfactory system is assembled during development and how it functions when mature.

A large body of evidence suggests that the olfactory receptors themselves contribute to glomerulus formation and placement in the mouse olfactory bulb (Mombaerts et al., 1996; Wang et al., 1998; Imai et al., 2006). In addition, several canonical axonal guidance cues and their receptors have also been shown to contribute to sensory axon targeting in the bulb (Renzi et al., 2000; Schwarting et al., 2000, 2004; Taniguchi et al., 2003; Cutforth et al., 2003; Miyasaka et al., 2005; Cho et al., 2007; Takeuchi et al., 2010). However, the precise sequence of cues that direct sensory axons to their targets has not yet been defined. The potential guidance roles of many candidate cues have not even been examined. In this study, we explored the role of Netrin/DCC (deleted in colorectal cancer) signaling in olfactory sensory axon guidance. Netrin 1 was one of the first axonal guidance cues to be identified (Hedgecock et al., 1990; Ishii et al., 1992), was the founding member of a small family of guidance cues (Serafini et al., 1994), and depending upon the receptors expressed by a responding axon, can act as either an attractant or a repellent (Hong et al., 1999).

In this study we define a role for Netrins in the guidance of a small group of olfactory sensory neurons that project to two spe- 
cific, identified locations in the olfactory bulb. We first generated lines of transgenic zebrafish in which the odorant receptor OR111-7 is expressed along with the transactivator Gal4. Gal4 expression in turn induced the expression of a fluorescent axonal tracer. The highly specific projection pattern of these labeled axons allowed us to analyze the effects of knocking down or misexpressing candidate guidance receptors and ligands.

Here we show that OR111-7 transgene-expressing neurons express the Netrin receptor DCC. Netrin la is expressed medially while netrin $1 b$ is detected ventrally in the bulb. Loss of Netrin/ DCC signaling induces ectopic projections of transgene-expressing axons both between the olfactory epithelium and the bulb and within the bulb itself. We show that Netrins act directly on olfactory sensory neurons. Our results are consistent with Netrins acting as attractants for a subset of olfactory sensory axons, drawing them both medially and ventrally. Thus, for the first time, Netrin/DCC signaling is shown to contribute to the initial targeting of olfactory sensory axons and to be required for the generation of a correct protoglomerular map in the olfactory bulb.

\section{Materials and Methods}

Transgenic zebrafish lines. Tg(omp:lyn-RFP) and Tg(trpc2:gap-VENUS)transgenic lines (hereby referred to as OMP:RFP and TRPC2:Venus, respectively) were obtained from the Yoshihara laboratory (Sato et al., 2005). The UAS:DCC-transgenic line was generated using the full-length DCC coding sequence with 7 of the first 27 base pairs mutated (ATGGGaTGtGTaACaGGcGAcATcCGC) so that it was resistant to the translation blocking morpholino we used in our experiments. This sequence was cloned into a Gateway middle clone, $\mathrm{pME}-\mathrm{dcc}_{\mathrm{m}}$, and confirmed by full-length sequencing ( $\operatorname{Lim}$ et al., 2011). The UAS:DCC transgenesis clone was built using the Tol2 kit (Kwan et al., 2007) and multisite Gateway (Invitrogen) recombination reactions. p5E-10xUAS, pME- $\mathrm{dcc}_{\mathrm{m}}$, and $\mathrm{p} 3 \mathrm{E}-\mathrm{pA}$ were recombined into pDestTol2CG2, which provided Tol2 transposon ends and a cmlc2:EGFP transgenesis marker. Injection of this DNA construct and generation of the stable transgenic line $\operatorname{Tg}\left(U A S: d c c_{m} ; m y l 7: E G F P\right)^{\text {zc79 }}$ was performed as described by Bonkowsky et al. (2008).

The following constructs were cloned into the mini-Tol2 vector (Balciunas et al., 2006) and injected into single-celled zebrafish embryos which were raised to adulthood (Fisher et al., 2006). Two or more independent founders were obtained for all transgenic lines made with the following constructs. (1) $\mathrm{Tg}$ (omp:GAL4), henceforth referred to as OMP:Gal4: The $2.1 \mathrm{~kb}$ olfactory marker protein (OMP) promoter, obtained from the Yoshihara laboratory (Sato et al., 2005), was cloned upstream from a Gal4 coding sequence. (2) $\mathrm{Tg}$ (orR111-7:IRES:GAL4), henceforth referred to as OR111-7: IRES:Gal4: We designed our transgenic approach based on studies that were pioneered in the mouse (Vassalli et al., 2002; Bozza et al., 2009). In our construct (depicted in Fig. 1), OR111-7 expression is regulated by its endogenous promoter and the E15-1 enhancer element which promotes odorant receptor expression (Nishizumi et al., 2007). The entire $4 \mathrm{~kb}$ of genomic sequence between the coding sequences of OR111-7 and its $5^{\prime}$ upstream gene, OR111-8, were included in the construct $\left(111-55^{\prime}\right)$. An IRES:Gal4 cassette was inserted after the OR111-7 coding sequence. This was followed by $1 \mathrm{~kb}$ of untranslated genomic sequence downstream from the OR111-7 coding sequence (OR111-7 3'). (3) Tg(UAS:gap43-CITRINE), hereafter referred to as UAS:gap43-Citrine: Fourteen tandem UAS sequences were used to drive the expression of a citrine fluorophore that was fused with the first 20 amino acids of the GAP43 protein to promote axon labeling.

DCC mutant. A DCC mutant was isolated by an ENU-induced screen (Granato et al., 1996), and mutant embryos of either sex were identified using standard PCR genotyping (R. A. Jain and M. Granato, in preparation).

Immunohistochemistry. Three-day-old zebrafish larvae were fixed overnight in $4 \%$ paraformaldehyde in $0.1 \mathrm{~m}$ phosphate buffer. Larvae were treated with acetone for $20 \mathrm{~min}$ at $-20^{\circ} \mathrm{C}$ to facilitate tissue permeabilization. Goat anti-GFP (1:100, Rockland Immunochemicals, catalog \#600-101-215) and anti-goat IgG Alexa Fluor 488 (1:500; Invitrogen) were used to visualize GFP-positive neurons. Propidium iodide staining was performed using the protocol of Brend and Holley (2009) with the omission of the RNase treatment step. Larvae were mounted face down or ventral side down to visualize projections along the dorsoventral axis or anteroposterior axes respectively. Larvae were imaged using a $40 \times$ oil-immersion lens on an inverted Leica TSP2 confocal microscope. Sections of $1 \mu \mathrm{m}$ thickness were taken through the entire olfactory bulb.

Whole-mount fluorescent in situ hybridization. Antisense digoxigeninlabeled RNA probes for DCC, netrin 1a, or netrin $1 \mathrm{~b}$ were generated using plasmids obtained from the Chien laboratory (Fricke and Chien, 2005; Suli et al., 2006). In situ hybridization was performed as described previously (Chalasani et al., 2007). In situ signals were amplified using a cyanine 5-coupled tyramide system (TSA Plus cyanine 5 System, PerkinElmer, product number NEL745001KT). In situ hybridization was followed by immunohistochemistry using goat anti-GFP (1:100, Rockland Immunochemicals, catalog \#600-101-215) and anti-goat IgG Alexa Fluor 488 (1:500; Invitrogen) to visualize axons in the context of RNA expression. This was followed by propidium iodide staining (as above). Processed larvae were mounted in the frontal or ventral orientation and imaged using a $40 \times$ oil-immersion lens on an inverted confocal microscope. Images were captured every micrometer through the entire extent of the olfactory bulb.

Morpholino injections into zebrafish embryos. Morpholinos (MOs) were obtained from Gene Tools. They were suspended in autoclaved water at a final stock concentration of $5 \mathrm{~mm}(\sim 41.5 \mathrm{ng} / \mathrm{nl})$. Male and female transgenic adult zebrafish were mated together to generate embryos of either sex for morpholino injection. Morpholinos were injected into the embryo yolk immediately after fertilization at the one cell stage. Morpholino sequences were identical to those used by Suli et al. (2006). Either DCC MO1, DCC MO2, netrin1a MO, or netrin1b MO (9 ng/ embryo) was injected along with $5 \mathrm{ng}$ of p53 morpholino (Robu et al., 2007). The p $53 \mathrm{MO}$ was routinely added to reduce cell death occurring as a side effect of morpholino treatment. For experiments using subthreshold morpholino doses, we first injected $4 \mathrm{ng} / \mathrm{embryo}$ of either netrin 1 a or netrin $1 \mathrm{~b}$ morpholino along with $5 \mathrm{ng}$ of $\mathrm{p} 53$ morpholino. A subset of the netrin morphants was immediately injected with $4 \mathrm{ng} / \mathrm{embryo}$ of DCC MO1 along with 5 ng of p53 MO. Using the same injection needle, we then injected previously uninjected embryos with DCC MO1 along with $5 \mathrm{ng}$ of $\mathrm{p} 53 \mathrm{MO}$.

Quantification of targeting errors. Larvae were processed for immunohistochemistry and imaged using confocal microscopy as described previously. The number of larval olfactory bulbs with axonal projections to either individual protoglomeruli or non-protoglomerular regions (posterior, ventral, midline, lateral, or anterior) were counted. Fisher's exact test was used to determine whether the control group was statistically different from the experimental group. The graphs represent the percentage of larval olfactory bulbs with axonal projections to the specified locations.

\section{Results}

Two general classes of olfactory sensory neurons project to specific defined neuropilar regions in the olfactory bulb

The zebrafish olfactory epithelium contains two well characterized sensory neuron populations that can be defined based on their position in the epithelium, their morphologies, and their molecular characteristics (Sato et al., 2005; Hamdani and Døving, 2007). Ciliated olfactory sensory neurons have long dendrites and are located deep within the olfactory epithelium. They express classical main olfactory bulb-type odorant receptors along with OMP (Celik et al., 2002; Sato et al., 2005). Microvillous olfactory sensory neurons have shorter dendrites and are located more superficially within the epithelium. These neurons express V2R-type vomeronasal receptors and also the transient receptor potential channel 2 (TRPC2). The axons of these two classes of OMP- and TRPC2-expressing neurons project to nonoverlapping regions of the olfactory bulb (Sato et al., 2005). 

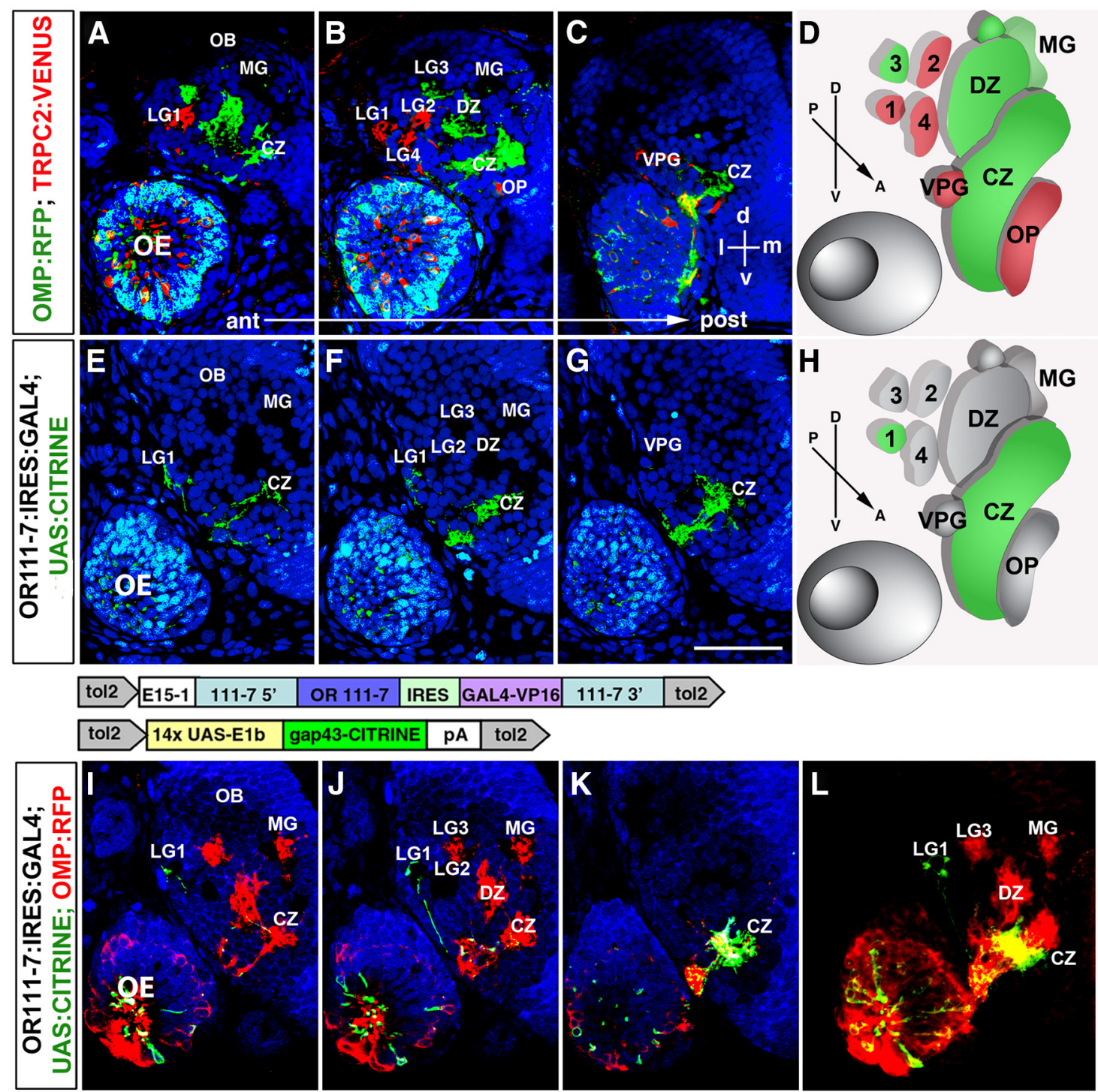

Figure 1. Olfactory sensory axons converge into distinct identifiable protoglomeruli in the 3-d-old zebrafish olfactory bulb. $\boldsymbol{A}-\boldsymbol{C}, \boldsymbol{E}-\mathbf{G}, \boldsymbol{I}-\boldsymbol{K}$, Single confocal sections through a $3-\mathrm{d}$-old olfactory bulb (frontal view). The absence of propidium iodide staining (blue) delineates distinct protoglomeruli in single sections along the anteroposterior axis. A-D, In OMP:RFP; TRPC2:Venus double transgenics, OMP:RFP (green)-labeled sensory axons project to the central zone (CZ), DZ, MG, and LG3 protoglomeruli while TRPC2:Venus (red)-labeled axons project to the ventral posterior glomerulus (VPG), olfactory plexus (OP), and LG1, LG2, and LG4 protoglomeruli. E-H, OR111-7:IRES:Gal4; UAS:Citrine-expressing axons primarily target the CZ protoglomerulus. Some OR111-7 transgene-expressing axons project to the LG1 protoglomerulus. I-K, Larvae harboring OR111-7:IRES:Gal4, UAS:Citrine, and OMP:RFP transgenes are shown. 0R111-7:IRES:Gal4; UAS:Citrine-expressing axons that target the CZ protoglomerulus also express OMP. L, Average intensity projection of serial confocal optical sections through the transgenic larva shown in $\mathbf{I} \boldsymbol{K}$. Scale bar (in G): $\boldsymbol{A}-\mathbf{C}, \mathbf{E}-\mathbf{G}, \mathbf{I}-\mathbf{L}, 50 \mu \mathrm{m}$.

By $3 \mathrm{~d}$ postfertilization ( $3 \mathrm{dpf}$ ), OMP and TRPC2 projections have coalesced into distinct protoglomeruli (Fig. $1 A-D$; Sato et al., 2005). Protoglomeruli are specialized neuropilar regions comprised of olfactory sensory axons and the dendrites of olfactory bulb neurons (Li et al., 2005). Since they are the only acellular regions in the olfactory bulb, they can be demarcated using cellular dyes such as BODIPY that label cells within the bulb while leaving protoglomeruli unlabeled (Dynes and Ngai, 1998). Individual protoglomeruli identified in this manner are stereotyped and consistently recognizable between larvae. In this study, we have used the protoglomerular nomenclature proposed by Dynes and Ngai (1998).

We used propidium iodide instead of BODIPY to label cells (presented in the blue color channel) and were able to identify 12 protoglomeruli as unstained regions in the olfactory bulb surrounded by olfactory bulb cells (Fig. 1). Using OMP:RFP and
TRPC2:Venus double-transgenic animals in combination with propidium iodide staining, we have identified the specific protoglomeruli that are targeted by either OMP- or TRPC2-expressing axons in 3-d-old olfactory bulbs (Fig. $1 D$; schematized in Fig. $1 A-C)$. OMP-expressing neurons (green) project to the central zone (CZ), dorsal zone (DZ), and lateral glomerulus 3 (LG3). A few OMP-expressing axons also innervate the medial glomeruli 1-4 (MG1-4). TRPC2-expressing neurons (red) target the olfactory plexus, lateral glomeruli 1, 2, and 4 (LG1, LG2, LG4), and the ventral posterior glomerulus. This pattern of targeting is repeatable from fish to fish, demonstrating that the axons of specific subsets of olfactory neurons target particular protoglomeruli. Aside from the small OMP projection, the MG protoglomeruli are incompletely filled by axons labeled in the OMP and TRPC2 transgenics. It is possible that the MG protoglomeruli receive additional axonal input from other less well characterized classes 
of olfactory sensory neurons, perhaps the recently identified TAAR- and/or V1R-expressing neurons (Saraiva and Korsching, 2007; Hussain et al., 2009).

\section{Construction of a transgenic line that expresses OR111-7 under the control of its flanking control sequences}

The study of axonal targeting in the zebrafish system has been impeded by an inability to visualize the axonal projections of sensory neurons that express a single odorant receptor. Inspired by the transgenic approach pioneered in the mouse by Vassalli et al. (2002), we generated two independent transgenic zebrafish lines in which a specific odorant receptor, OR111-7, is expressed along with a reporter under the control of its flanking non-coding sequences. We expected that transgene-expressing sensory neurons would project axons to a single specific protoglomerulus. We chose to study OR111-7 because its expression is detected $\sim 30 \mathrm{~h}$ postfertilization (hpf) when some of the earliest axons are extending into the olfactory bulb (Barth et al., 1997). Our construct is schematized in Figure 1 (middle) and is composed of the following elements: (1) the E15-1 enhancer element identified by Nishizumi et al. (2007) that is near the OR111 gene family cluster and acts in cis- to promote odorant receptor expression, (2) $4 \mathrm{~kb}$ of genomic sequence upstream to the OR111-7 coding sequence (OR 5'), (3) The OR111-7 coding sequence, (4) an IRES sequence followed by a sequence encoding the transactivating factor Gal4VP16, and (5) $1 \mathrm{~kb}$ of genomic sequence located just 3 ' to the OR111-7 coding region. This construct links olfactory receptor expression to the simultaneous expression of the transactivating factor Gal4. Two independent transgenic lines were made with this construct and appear equivalent in their properties.

OR111-7:IRES:Gal4-transgenic fish were mated with fish containing a UAS driven Gap43-Citrine transgene. The fluorescent fusion protein is transported into axons and brightly labels axonal projections. The progeny that contain both transgenes have a small number of labeled cells that are restricted exclusively to the olfactory epithelium. As would be expected, fluorescently labeled cells are also labeled in situ with a probe for OR111-7 (data not shown). They elaborate axons that extend into the olfactory bulb. OR111-7 transgene-expressing neurons are first detected $\sim 26-27 \mathrm{hpf}$. Surprisingly, we observe that the number of labeled transgene-expressing neurons decreases with developmental age. At $3 \mathrm{dpf}$, an average of $\sim 27$ neurons in each olfactory pit $(n=30$ pits) express the OR111-7 transgene, but only 13 neurons in $7 \mathrm{dpf}$ larvae ( $n=14$ pits), and 6 neurons in 9 dpf larvae ( $n=4$ pits) express the transgene. The decline in transgene-expressing neurons suggests that it may be inactivated over time. It is possible that regulatory elements required for continued stable expression of the odorant receptor are missing in our transgene construct. Alternatively, the expression of endogenous odorant receptors may suppress the expression of the transgenic odorant receptor through processes analogous to those that limit odorant receptor expression to a single allele in each sensory neuron (Shykind, 2005).

The axons of OR111-7 transgene-expressing olfactory sensory neurons project to the central zone protoglomerulus and sometimes to the LG1 protoglomerulus

At $3 \mathrm{dpf}$ the majority of axons extending from transgeneexpressing neurons converge within the bulb upon the central zone protoglomerulus (Fig. $1 E-H$ ). This is reminiscent of a previous study in which the OR111-7 coding sequence was replaced with a YFP reporter in a BAC-transgenic animal and the resulting YFP-labeled axons were shown to target a ventromedial area likely to be the central zone protoglomerulus (Sato et al., 2007). The central zone protoglomerulus is large and ventrally positioned with its longest axis oriented anterior-posteriorly. It is thought to be composed of approximately five smaller "sub"protoglomeruli (Dynes and Ngai, 1998). At 3 dpf, OR111-7 transgene-expressing axons are not localized to any specific subcompartment within the central zone. Rather, axons are distributed and appear to terminate throughout the whole anterior to posterior extent of the protoglomerulus. In $\sim 60 \%$ of larvae, a small number of OR111-7 transgene-expressing axons project to LG1. This was surprising since LG1 is normally innervated exclusively by TRPC2- and V2R-type receptor-expressing axons (Fig. $1 A-D)$. To examine whether OR111-7-expressing axons are ciliated olfactory sensory neurons, we crossed the OR111-7:Gal4; UAS:Citrine transgenics with OMP:RFP transgenics and examined fish with all three transgenes. The Citrine and mRFP fluorescent marker proteins are fused with membrane targeting regions of the GAP43 and lyn-tyrosine kinase respectively. As a result, these fluorescent reporter proteins are differentially distributed in neurons. Thus, while OR111-7-dependent Citrine expression colocalizes with OMP expression in axons, it is difficult to observe overlapping expression in the cell bodies. We found that all of the axons expressing both the OR111-7 and OMP transgenes project to the central zone, suggesting that these OR111-7expressing cells are ciliated olfactory sensory neurons (Fig. 1I-L). A small number of sensory neurons expressing the OR111-7 transgene but not the OMP:RFP transgene project to LG1 (Fig. $1 E, F, I, J, L$; see Figs. $4 A, D, G, J, 5 A, E, M, 7 A, 8 A, E, 10 A)$. These results show that the OR111-7 transgene is largely expressed in OMP-type neurons that project exclusively to the central zone. We speculate that the OR111-7-expressing neurons that extend to LG1 express TRPC2 and belong to the V2R type of olfactory sensory neurons.

Since the predominant projection of OR111-7 transgeneexpressing neurons is to the central zone, these transgenic fish are a valuable tool for testing the roles of candidate guidance cues in targeting axons to a consistent and identifiable location in the developing olfactory bulb. In this study we assessed the contribution of Netrin/DCC signaling in properly targeting axons to the central zone.

\section{DCC is expressed in OMP- and OR111-7 transgene-expressing olfactory sensory neurons}

OMP:Gal4-expressing olfactory sensory axons (green, Fig. $2 A, C$ ) have entered the primordial olfactory bulb in $24 \mathrm{hpf}$ embryos. At this time, there are on average of 24 labeled olfactory sensory neurons in each olfactory pit and $\sim 75 \%$ of them express DCC mRNA (red, arrowheads in Fig. $2 C ; N=241$ neurons from 8 olfactory pits). A high level of DCC expression is also detected in olfactory bulb cells (Fig. 2A, arrow). The axons of OR111-7 transgene-expressing neurons (green, Fig. $2 B, D$ ) enter the bulb by $27 \mathrm{hpf}$. At this age, there are on average 8 labeled olfactory sensory neurons in each olfactory pit and $\sim 87 \%$ of them express DCC mRNA (red, arrowheads in Fig. $2 D ; N=87$ neurons from 11 olfactory pits). At $53 \mathrm{hpf}$, when axons have already reached the presumptive central zone, there are on average 23 Citrine-labeled OR111-7 transgene-expressing neurons and $\sim 35 \%$ of them express DCC mRNA (data not shown, $N=232$ neurons from 10 olfactory pits). These results indicate that the Netrin receptor DCC is expressed in OMP-expressing sensory neurons and in most OR111-7 transgene-expressing neurons early as their axons are growing into the olfactory bulb. 


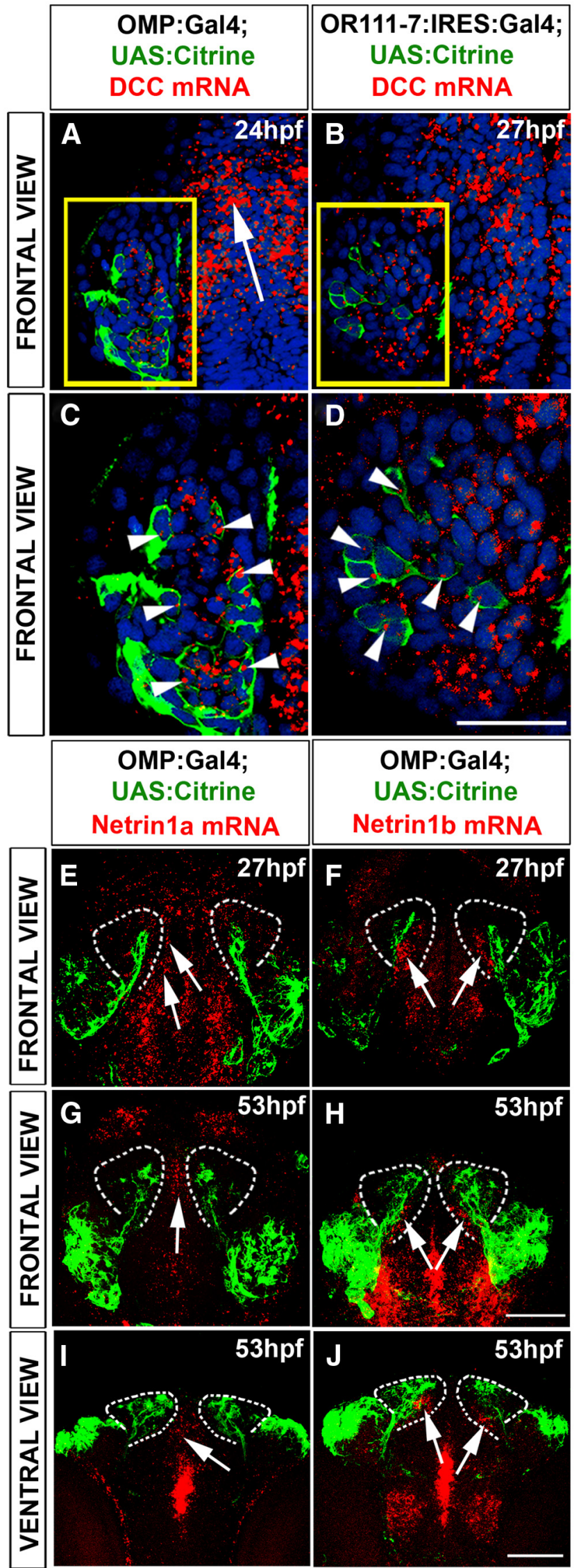

Figure 2. DCC mRNA is expressed in OR111-7 transgene-expressing neurons, netrin1a mRNA is expressed at the telencephalic midline, and netrin $1 \mathrm{~b}$ mRNA is expressed ventrally within the olfactory bulb. $\boldsymbol{A}, \boldsymbol{B}$, Propidium iodide (blue) demarcates cells in the
Netrin $1 \mathrm{a}$ is expressed at the telencephalic midline and netrin $1 b$ is expressed ventrally in the olfactory bulb

Netrin $1 \mathrm{a}$ and netrin $1 \mathrm{~b}$ expression patterns were examined at $27 \mathrm{hpf}$ when olfactory axons are extending into the bulb, and at $53 \mathrm{hpf}$ when they have navigated into the central zone (Fig. 2). Netrin mRNA expression was visualized along the dorsoventral axis (in frontal views) and along the anteroposterior axis (in ventral views). At 27 and $53 \mathrm{hpf}$, netrin la mRNA (red) is expressed in a dense strip of cells near the margin of the bulb at the telencephalic midline (Fig. $2 E, G$, arrow) all along the anteroposterior axis (Fig. 2I, arrow; data not shown). At 27 and $53 \mathrm{hpf}$, netrin $1 \mathrm{~b}$ mRNA (red) is present more ventrally in close proximity to where olfactory sensory axons enter the bulb (green, arrows in Fig. $2 F, H, J)$. These findings suggest that these two secreted Netrins are expressed at a time and in a place where they could influence the trajectories of olfactory sensory axons.

Sensory neurons expressing OMP, but not TRPC2, display pathfinding errors in DCC morphants

A majority of OMP:Gal4; UAS:Citrine neurons express the DCC receptor by 24 hpf as axons extend into the olfactory bulb. Fluorescent TRPC2:Venus neurons are first detected $\sim 42 \mathrm{~h}$ postfertilization, when axons have already entered the olfactory bulb. DCC mRNA is not detected in TRPC2 neurons at this age (data not shown). However, it is possible that DCC is expressed earlier than $42 \mathrm{hpf}$ in immature neurons that will express TRPC2

$\leftarrow$

olfactory epithelium in a frontal view of zebrafish embryos. $A$, $B$, By $24 \mathrm{hpf}, \mathrm{DCC} \mathrm{mRNA}$ (red) is expressed in both the olfactory epithelium (boxed) and in the nascent olfactory bulb (white arrow). The boxed regions are magnified in $\boldsymbol{C}$ and $\mathbf{D}$. C, DCC mRNA (red, arrowheads) is detected in 0MP:Gal4; UAS:Citrine (green) neurons at $24 \mathrm{hpf}$ as axons first extend into the olfactory bulb. B, D, At $27 \mathrm{hpf}$, most 0R111-7 transgene-expressing neurons (green) express DCC mRNA (red, arrowheads). $\boldsymbol{E}-\boldsymbol{J}$, Maximum intensity projections of serial confocal optical sections through OMP:Gal4; UAS:Citrine-transgenic embryos. $\boldsymbol{E}-\boldsymbol{H}$, In frontal views, dorsal is to the top and ventral toward the bottom of the image. $I, J$, In ventral views, anterior is to the top and posterior toward the bottom. $\mathbf{E}, \mathbf{G}, \mathbf{I}$, At both 27 and 53 hpf, Netrin1a mRNA (red) is detected at the telencephalic midline along the anteroposterior axis of the olfactory bulb (arrows). $\boldsymbol{F}, \boldsymbol{H}, \boldsymbol{J}$, Netrin $1 \mathrm{~b}$ mRNA is expressed within the ventral region of the olfactory bulb (arrow), close to the presumptive central zone at 27 and $53 \mathrm{hpf}$. The olfactory bulb is outlined with dashed lines. Scale bar (in D): $\boldsymbol{A}, \boldsymbol{B}, 50$ $\mu \mathrm{m}, \boldsymbol{C}, \boldsymbol{D}, 25 \mu \mathrm{m}$. Scale bar (in $\boldsymbol{H}$ ): $\boldsymbol{E}-\boldsymbol{H}, 50 \mu \mathrm{m}$. Scale bar (in J): $I, J, 50 \mu \mathrm{m}$. 


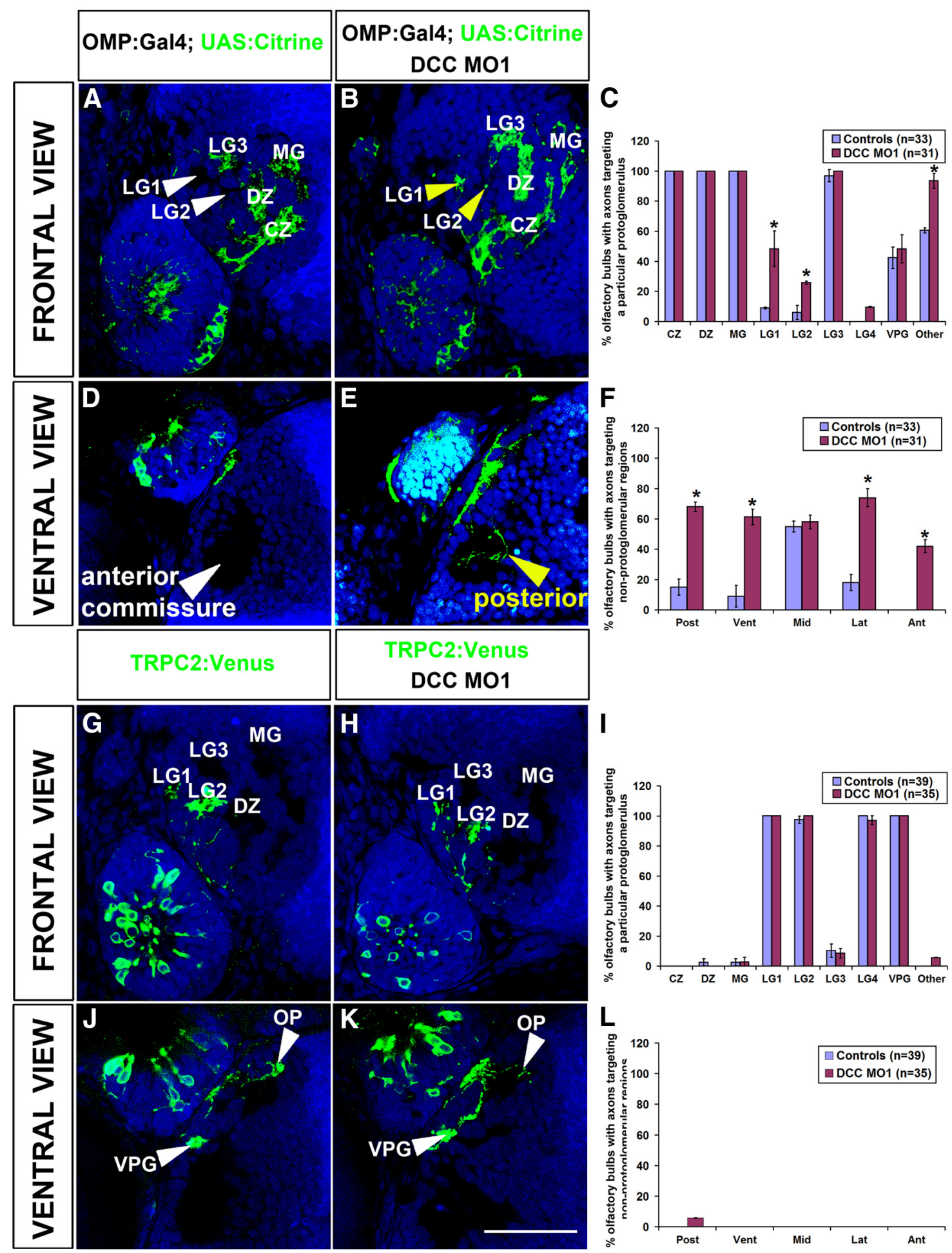

Figure 3. Sensory neurons expressing OMP, but not TRPC2, display pathfinding errors in DCC morphants. $A, B, D, E$, Single confocal optical sections through 3-d-old OMP:Gal4; UAS:Citrine larvae. $\boldsymbol{G}, \boldsymbol{H}, \boldsymbol{J}, \boldsymbol{K}$, Single confocal optical sections through 3-d-old TRPC2:Venus larvae. Propidium iodide (blue) allows the identification of distinct protoglomeruli. $\boldsymbol{C}, \boldsymbol{F}, \boldsymbol{I}, \boldsymbol{L}$, The percentages of olfactory bulbs displaying a particular guidance error are shown. Embryos injected with DCC MO1 (purple bars) are compared with sibling control larvae (blue bars). Fisher's exact test was used to test for statistical significance, $p<0.05$. Error bars indicate the SEM. $A, C$, In control larvae, OMP:Gal4; UAS:Citrine axons do not enter LG1 or LG2 (white arrowheads). $B, C$, Knocking down DCC levels causes axons to inappropriately enter LG1 and LG2 (yellow arrowheads). $\boldsymbol{D}, \boldsymbol{F}$, Control OMP-expressing axons enter the olfactory bulb and do not misproject posteriorly into the anterior commissure (white

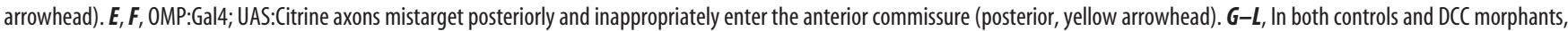
TRPC2-expressing axons innervate LG1, LG2, LG4, VPG and OP. No guidance errors are observed upon DCC inhibition. Scale bar (in $\boldsymbol{K}$ ): $\boldsymbol{A}, \boldsymbol{B}, \boldsymbol{D}, \boldsymbol{E}, \mathbf{G}, \boldsymbol{H}, \boldsymbol{J}, \boldsymbol{K}, 50 \mu \mathrm{m}$. CZ, Central zone; VPG, ventral posterior glomerulus; $\mathrm{OP}$, olfactory plexus; Other, axonal projections to non-protoglomerular regions of the olfactory bulb; Post, posterior; Vent, ventral; Mid, midline; Lat, lateral; Ant, anterior. These individual categories are grouped together as 0 ther in $\boldsymbol{C}$ and $\boldsymbol{I}$. 

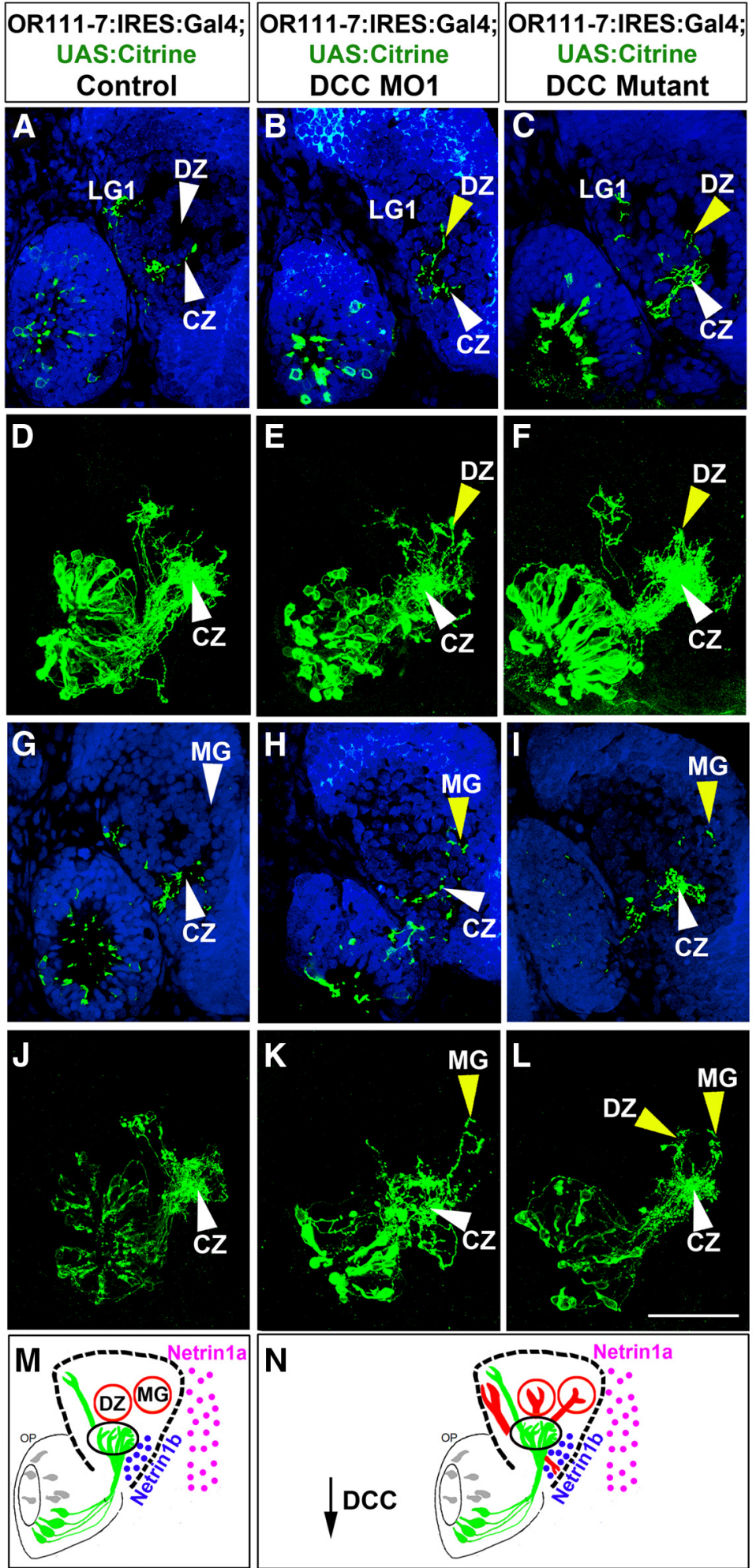

Figure 4. OR111-7transgene-expressing sensory neurons misproject into dorsal protoglomeruli in DCC morphants and in a DCC mutant. $\boldsymbol{A}-\boldsymbol{C}, \mathbf{G}-\mathbf{I}$, Single confocal optical sections through 3-d-old OR111-7:IRES:Gal4; UAS:Citrine larvae (frontal view). Dorsal is to the top and the midline is to the right of the image. Propidium iodide (blue) allows the identification of distinct protoglomeruli. $\boldsymbol{D}-\boldsymbol{F}, \boldsymbol{J}-\boldsymbol{L}$, Maximum intensity projections of serial confocal optical sections from the same larvae shown immediately above. $\boldsymbol{M}, \boldsymbol{N}$, Schematics showing control OR111-7:IRES:Gal4; UAS:Citrine projections (green) and mistargeted axons (red) observed upon DCC knockdown. The thickness of the red lines corresponds roughly to the penetrance of the indicated phenotypes. Netrin1a mRNA (pink dots) is expressed at the telencephalic midline and netrin $1 \mathrm{~b}$ mRNA (blue dots) is expressed in the ventral bulb. Black circle, central zone; Red circles, dorsal zone, medial glomeruli. $\boldsymbol{A}, \mathbf{D}, \mathbf{G}, \boldsymbol{J}, \boldsymbol{M}$, In uninjected embryos, 0R111-7 transgene-expressing axons (green) target the central zone (CZ, white arrowhead) and LG1. $\boldsymbol{A}, \boldsymbol{M}$, Control OR111-7-transgenic axons do not enter DZ (white later. To examine whether DCC is required for proper axonal pathfinding, we used a translation blocking morpholino, DCC MO1, previously characterized by Suli et al. (2006) to reduce DCC levels specifically in OMP- or TRPC2-expressing neurons. Embryos obtained by mating transgenic OMP:Gal4 zebrafish with UAS: Citrine transgenics or TRPC2:Venus transgenics with wild-type animals were injected with DCC MO1 at the single-cell stage. Fluorescent larvae were fixed at $3 \mathrm{~d}$ postfertilization and processed for immunohistochemistry and propidium iodide staining. Uninjected controls were compared with morpholino-injected larvae (morphants) in frontal views to examine axonal projections along the dorsoventral axis and in ventral views to observe axons along the anteroposterior axis. Importantly, all protoglomeruli were easily discernable in 3-d-old DCC morphants and had their characteristic morphologies in their expected locations. Thus, the DCC morpholino did not have a observable effect upon protoglomerular development. Since it was impossible to quantify the number of individual axons making a particular targeting error (severity of the phenotype), instead we quantified the number of olfactory bulbs in which a specific axonal guidance error was observed (penetrance of the phenotype). This is done by assigning a score, 0 for absence and 1 for the presence of a particular phenotype. We then counted the total number of bulbs with a given phenotype and express this as a percentage (Fig. 3C, F, I,L). Reducing DCC levels in OMP-expressing neurons causes their axons to inappropriately target the lateral glomeruli LG1 and LG2 (Fig. $3 A-C$ ). In addition, in $\sim 70 \%$ of DCC morphants, OMP-expressing axons ectopically project posteriorly, away from the bulb, instead of entering it (Fig. 3D-F). In contrast, TRPC2-expressing neurons have no detectable axon guidance errors upon DCC knockdown (Fig. 3G-L). These data show that DCC-mediated signaling is specifically required for the pathfinding of axons of OMP but not TRPC2-expressing olfactory neurons.

arrowhead). $\boldsymbol{B}, \boldsymbol{E}, \boldsymbol{C}, \boldsymbol{F}, \boldsymbol{N}$, In embryos injected with DCC M01 or in DCC mutants, OR111-7-transgenic axons target the $C Z$ (white arrowheads), but also inappropriately enter the $D Z$ (yellow arrowheads). G, M, Uninjected control OR111-7 transgene-expressing axons do not enter the MG (white arrowhead). $\boldsymbol{H}, \boldsymbol{I}, \boldsymbol{K}, \boldsymbol{L}, \boldsymbol{N}, \mathbf{O R} 111-7$ transgene-expressing axons misproject into MG and DZ protoglomeruli in DCC morphants and DCC mutant larvae (yellow arrowheads). Scale bar (in $\boldsymbol{L}$ ): A-L, $50 \mu \mathrm{m}$. 


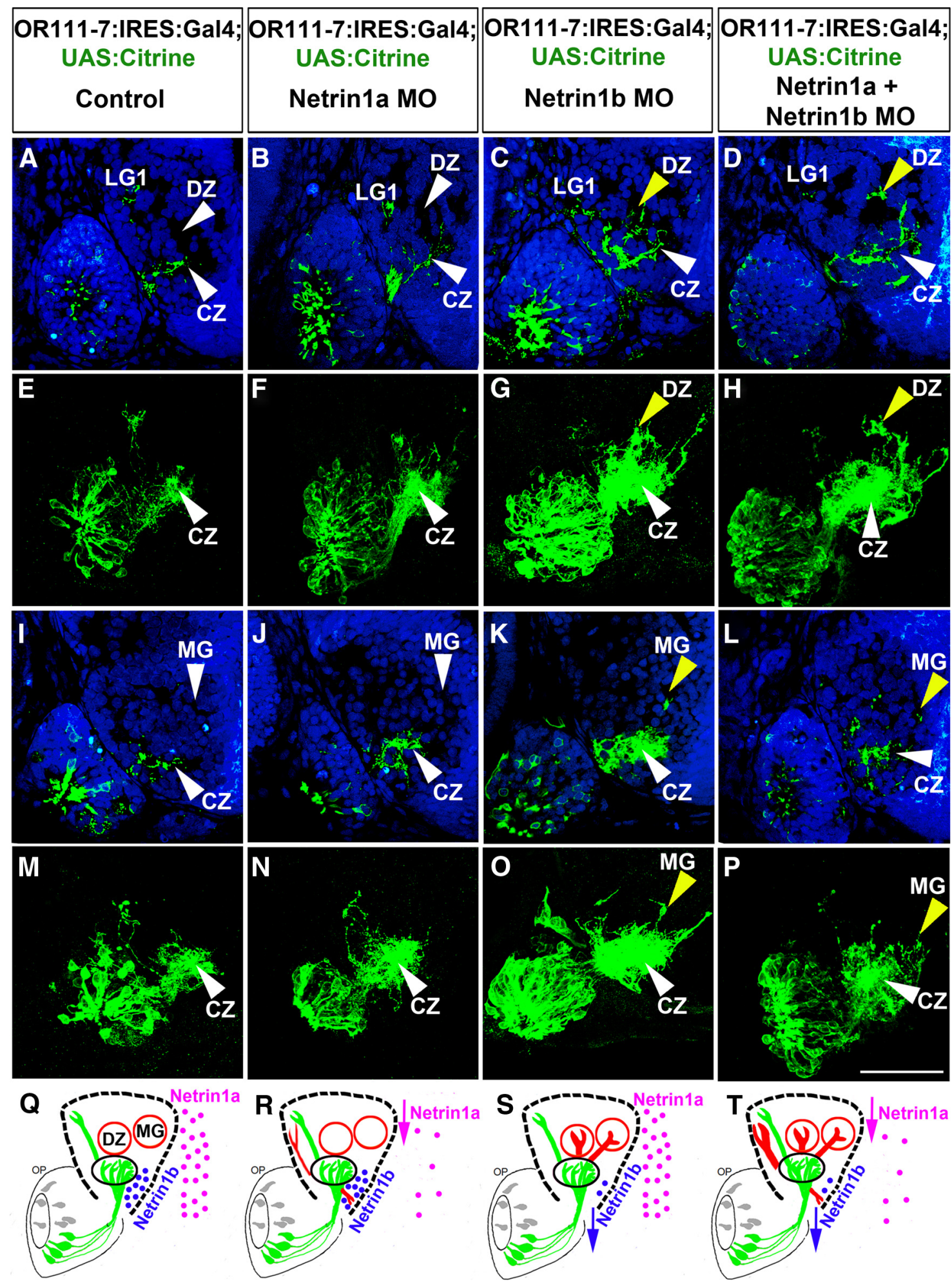

Figure 5. Inhibiting netrin $1 b$, but not netrin1a, phenocopies the dorsal protoglomerular misprojections of OR111-7-transgenic axons observed in DCC morphants. $A-D, I-L$, Single confocal optical sections through 3-d-old OR111-7:IRES:Gal4; UAS:Citrine larvae (frontal view). Dorsal is to the top and midline to the right of the image. Propidium iodide (blue) delineates distinct protoglomeruli in the olfactory bulb. $\boldsymbol{E}-\boldsymbol{H}, \boldsymbol{M}-\boldsymbol{P}$, Maximum intensity projections of serial confocal optical sections of the same larvae shown immediately above. $\mathbf{Q}-\boldsymbol{T}$, Schematics showing control OR111-7:IRES:Gal4; UAS:Citrine projections (green) and mistargeted axons (red) observed upon reducing Netrin1a or Netrin 1b levels. The thickness of the red lines corresponds roughly to the penetrance of the indicated phenotypes. Netrin 1a mRNA (pink dots) is expressed at the telencephalic midline and netrin 1b (blue dots) is expressed in the ventral bulb. Black circle, central zone; Red circles, dorsal zone, medial glomeruli. $A, E, I, M, Q$, Uninjected control OR111-7 transgene-expressing axons (green) project to the central zone (CZ, white arrowhead) and LG1.A, B, Q, R, OR111-7 transgene-expressing axons do not enter the $D Z$ (white arrowhead) in uninjected control embryos or in netrin1a morphants. C, G, D, H, S, $\boldsymbol{T}$, Knocking down netrin1b alone or netrin1a and netrin $1 \mathrm{~b}$ together causes OR111-7 transgene-expressing axons to inappropriately enter the DZ (yellow arrowheads). I, J, Q, R, OR111-7:IRES:Gal4; UAS:Citrine axons do not enter the MG (white arrowheads) in controls or in netrin1a morphants. $K, \mathbf{O}, \mathbf{L}, \mathbf{P}, \mathbf{S}, \boldsymbol{T}$, Reducing the level of netrin $1 \mathrm{~b}$ alone or netrin1a and netrin $1 \mathrm{~b}$ together causes axonal misprojections into $\mathrm{MG}$ (yellow arrowheads). Scale bar (in P): A-P, $50 \mu \mathrm{m}$. 


\section{OR111-7 transgene-expressing sensory neurons misproject into dorsal protoglomeruli in DCC morphants and in a DCC mutant}

OR111-7-expressing neurons are a subset of OMP-expressing neurons that express the DCC receptor and primarily target a single protoglomerulus. This allowed us to investigate in detail the contribution of DCC signaling toward axonal targeting within the zebrafish olfactory bulb. We first examined whether reducing DCC expression affects the protoglomerular targeting of OR111-7 transgene-expressing axons. Two separate approaches were used to decrease DCC levels in vivo. First, OR111-7:IRES:Gal4 and UAS:Citrine transgenic fish were crossed together. The resulting embryos were injected with one of two different translation blocking morpholinos, DCC MO1 or DCC MO2, both of which were previously characterized by Suli et al. (2006). OR111-7:IRES:Gal4; UAS:Citrine double-transgenic larvae with fluorescent olfactory sensory neurons were identified and processed for immunohistochemistry and propidium iodide staining. Axonal projections of uninjected controls were compared with larvae that were injected with morpholinos (morphants). There was no difference in the number of OR111-7-expressing neurons in controls (an average of $27 \pm 1$ neurons per olfactory pit, $n=30$ pits) and DCC morphants (an average of $26 \pm 2$ neurons per olfactory pit, $n=21$ pits) suggesting that there was no nonspecific cell death due to morpholino injections. Second, we examined the projections of OR111-7-transgenic axons in larvae with a hypomorphic DCC allele that has a missense mutation in the DCC coding sequence (Granato et al., 1996; Jain and Granato, in preparation). Adult OR111-7: Gal4; UAS:Citrine double transgenics that carried a single DCC mutant allele were generated. Mating these transgenics with their siblings generated fluorescent progeny of which 25\% were also homozygous mutant for DCC. Genomic DNA was extracted from the tails of 3-d-old fluorescent larvae to test for the presence of the DCC mutant allele while their heads were processed for immunohistochemistry. Axonal projections in individual mutant larvae were compared with siblings that carried wild-type DCC alleles. In general, the axonal guidance phenotypes observed in morphant and mutant fish are similar but stronger in morphants. The guidance phenotypes we observed are quantified in Figure $6 A-C$. We quantified the number of olfactory bulbs in which a particular axonal guidance error is detected (penetrance of the phenotype) because of the difficulty in quantifying the number of individual axons making a particular targeting error (severity of the phenotype). As
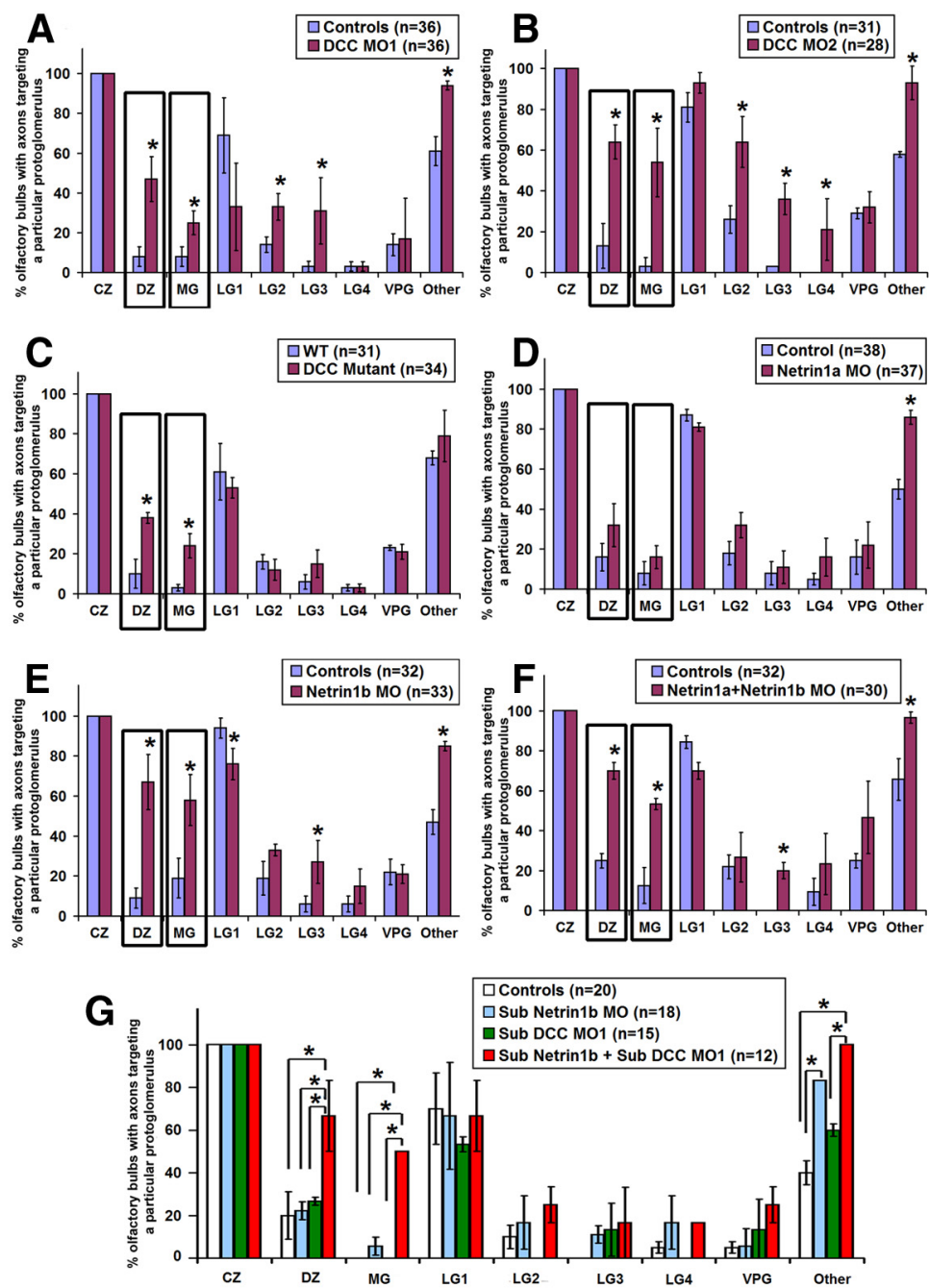

Figure 6. Quantification of protoglomerular targeting errors observed upon knocking down DCC, Netrin1a, Netrin 1b, or both Netrins together. $\boldsymbol{A}-\mathbf{G}$, The percentage of embryos with OR111-7:IRES:Gal4 axonal projections into the specified protoglomeruli is shown. Error bars indicate the SEM. A-C, Embryos injected with DCC M01, DCC M02, or DCC mutant larvae (purple bars) are compared with sibling control larvae (blue bars). $\boldsymbol{D}-\boldsymbol{F}$, Embryos injected with netrin $1 \mathrm{M} \mathbf{M} 0$, netrin $1 \mathrm{~b} M 0$, or netrin1a and netrin $1 b$ MOs together (purple bars) are compared with uninjected control siblings (blue bars). $G$, The axonal projections of uninjected controls (white bars), subthreshold netrin $1 \mathrm{~b}$ morphants (blue bars), and subthreshold DCC morphants (green bars); are compared with morphants injected with subthreshold doses of both netrin $1 \mathrm{~b}$ and $\mathrm{DCC}$ morpholinos together (red bars). $\boldsymbol{A}-\boldsymbol{C}$, The percentages of larval olfactory bulbs with 0R111-7-transgenic axonal projections to DZ and MG (boxed) significantly increase upon DCC knockdown using DCC M01, DCC M02, or in the DCC mutant. DCC M01 or M02 morphants also show increased misprojections into LG2 and LG3, while DCC mutant larvae do not have misprojections into any lateral protoglomeruli. $D-F, 0 R 111-7$-transgenic axons inappropriately enter DZ and MG at a statistically higher rate in netrin $1 b$ and in netrin $1 \mathrm{a}+$ netrin $1 \mathrm{~b}$ morphants, but not netrin 1a morphants. Inhibiting netrin $1 \mathrm{~b}$ alone or netrin $1 \mathrm{a}$ and netrin $1 \mathrm{~b}$ together also causes increased axonal projections to the LG3. G, Embryos injected with subthreshold doses of both netrin $1 b$ and DCC morpholinos display increased misprojections into DZ and $M G$, while embryos injected with subthreshold doses of either netrin $1 b$ or DCC morpholino alone do not misproject dorsally. $\boldsymbol{A}, \boldsymbol{B}, \boldsymbol{D}-\boldsymbol{F}$, Inhibiting DCC, netrin $1 \mathrm{a}$, netrin $1 \mathrm{~b}$, or both netrins together causes increased non-protoglomerular misprojections of 0R111-7-transgenic axons (0ther, described in detail in Figs. 7-9). Fisher's exact test was used to test for statistical significance, $p<0.05$. CZ, Central zone; VPG, ventral posterior glomerulus; Other, axonal projections to non-protoglomerular regions of the olfactory bulb.

explained earlier, this is done by assigning a score, 0 for absence and 1 for the presence of a particular phenotype. The total number of bulbs with a given phenotype are then counted and expressed as a percentage. The data are schematized in Figure $4 M, N$. Green axons indicate normal projections while red axons represent targeting errors. The thickness of the red line corresponds to the penetrance of the phenotype, and not its severity. In both DCC morphants and DCC mutant larvae, axons of OR111-7 transgene-expressing axons misproject into the $\mathrm{DZ}$ and $\mathrm{MG}$ 


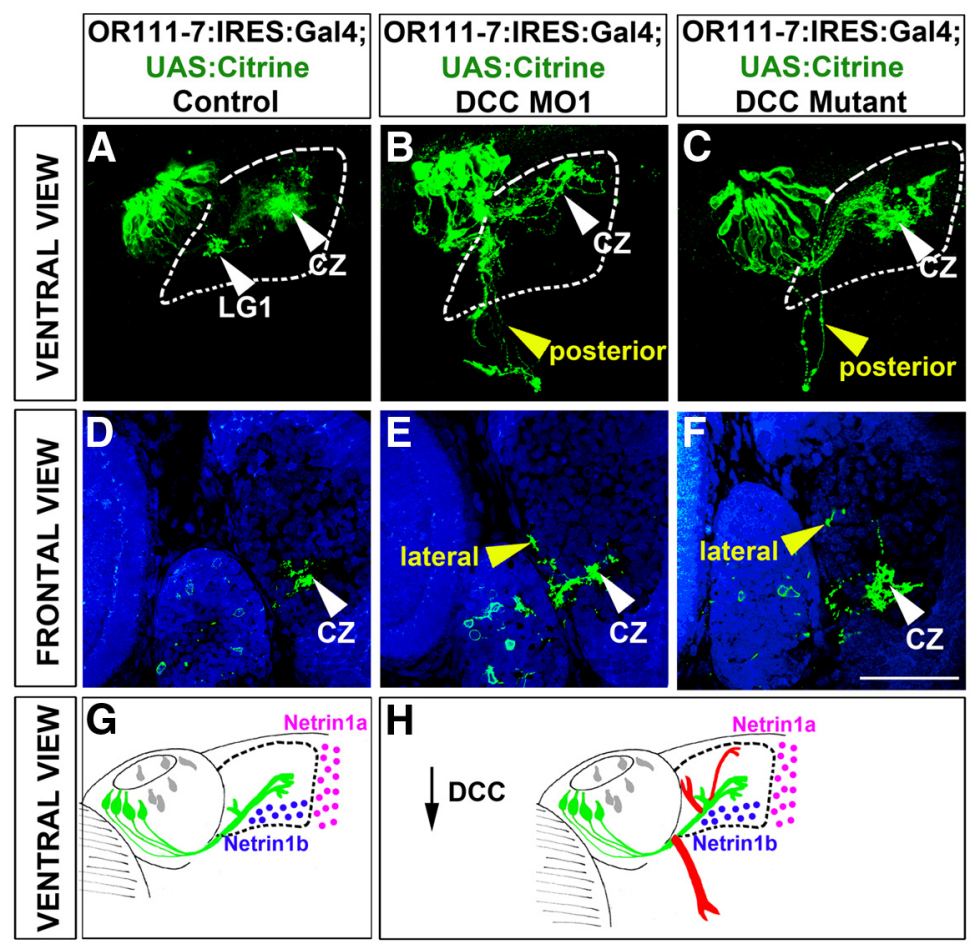

Figure 7. DCC signaling helps OR111-7 transgene-expressing axons enter the olfactory bulb and prevents nonprotoglomerular mistargeting within the bulb. $\boldsymbol{A}-\boldsymbol{C}$, Maximum intensity projections of serial confocal optical sections of 3-d-old larvae (ventral view). Anterior is toward the top and the midline to the right of the image. The olfactory bulb is outlined with dashed lines. $\boldsymbol{G}, \boldsymbol{H}$, Schematics showing control OR111-7:IRES:Gal4; UAS:Citrine projections (green) and mistargeted axons (red) observed upon inhibiting $D C C$. The thickness of the red lines corresponds roughly to the penetrance of the indicated phenotypes. Netrin1a mRNA (pink dots) is expressed at the telencephalic midline and netrin $1 \mathrm{~b}$ mRNA (blue dots) is expressed in the ventral bulb. $A, G, O R 111-7$ transgene-expressing axons (green) primarily target the central zone (CZ, white arrowhead) and LG1. $\boldsymbol{B}, \boldsymbol{C}, \boldsymbol{H}$, In DCC M01 morphants and in the DCC mutant, some 0R111-7 transgeneexpressing axons extend posteriorly away from the olfactory bulb and do not enter it (posterior, yellow arrowhead). $\boldsymbol{D}-\boldsymbol{F}$, Single confocal optical sections through frontally mounted 3-d-old OR111-7:IRES:Gal4; UAS:Citrine larvae. Propidium iodide (blue) delineates distinct protoglomeruli in the olfactory bulb. D, 0R111-7:IRES:Gal4; UAS:Citrine axons are detected in the central zone (CZ, white arrowhead). $\boldsymbol{E}, \boldsymbol{F}$, Reducing DCC levels via DCC M01 morpholino or in the hypomorphic DCC mutant causes some OR111-7-transgenic axons to mistarget toward the lateral border of the olfactory bulb posterior to the LG1 protoglomerulus (lateral, yellow arrowhead, schematized in Fig. $3 M, N$ ). Scale bar (in $\boldsymbol{F}$ ): $\mathbf{A}-\boldsymbol{F}, 50 \mu \mathrm{m}$.

protoglomeruli (Fig. $4 A-F, G-L, M, N$; quantified in Fig. $6 A-C$ ). These errors were incompletely penetrant, but consistent in all three experimental conditions. Projections to dorsal protoglomeruli are rare in control larvae. Additionally, DCC MO1 or MO2 morphants also show increased axonal misprojections into the lateral glomeruli LG2 and LG3 (see Fig. 6A,B). Since we observed similar misprojections using two different morpholinos to DCC and confirmed dorsal misprojections in the DCC mutant, we are confident that these errors are not caused by off target effects of the morpholinos or undefined genetic variation in the mutant fish.

OR111-7 transgene-expressing sensory neurons misproject into dorsal protoglomeruli in netrin $1 \mathrm{~b}$ morphants

We next tested whether decreasing levels of the likely ligands of the DCC receptor, netrin1a or netrin 1b, phenocopy the targeting errors induced in DCC knockdown fish. Netrinla or netrin $1 \mathrm{~b}$ were targeted with morpholinos previously characterized by Suli et al. (2006). Knockdown of netrin $1 \mathrm{~b}$ alone induces axonal misprojections of OR111-7 transgene-expressing neurons into both the DZ protoglomerulus (Fig. $5 A, C, E, G, Q, S$; quantified in Fig. $6 E$ ), and the MG protoglomerulus (Fig. 5I, $K, M, O, Q, S$; quantified in Fig. $6 E$ ). This observation recapitulates the finding that mistargeting to these more dorsal protoglomeruli is greatly enhanced when DCC expression is reduced. To further examine the interaction between netrin $1 \mathrm{~b}$ and DCC, we injected "subthreshold" doses of netrin $1 \mathrm{~b}$ and DCC morpholinos (Fig. $6 G)$. Subthreshold knockdown of either netrin $1 b$ or DCC does not induce significant misprojections to the $\mathrm{DZ}$ or $\mathrm{MG}$ protoglomeruli. However, subthreshold doses of both netrin $1 \mathrm{~b}$ and DCC morpholinos together recapitulates the dorsal misprojections observed upon severely knocking down either netrin $1 \mathrm{~b}$ or DCC alone. In contrast, knockdown of netrin la did not induce any significant axonal mistargeting of OR111-7 transgene-expressing neurons to any protoglomeruli (Fig. $5 A, B, E, F, I, J, M, N, Q, R$; quantified in Fig. $6 D)$. Knockdown of both netrinla and netrin $1 b$ induced the same protoglomerular mistargeting as netrin $1 \mathrm{~b}$ knockdown alone (Fig. $5 A, D, E, H, I, L, M, P, Q, T$; quantified in Fig. $6 F)$. We presume that the netrinla morpholino is at least partially effective since its knockdown causes other guidance errors (see Fig. 9D; Fig. 8A, B,E,F,I$, J, M, N)$. These data suggest that netrin $1 \mathrm{~b}$ works together with DCC to help target the axons of OR111-7 transgene-expressing axons ventrally.

\section{Netrin/DCC signaling contributes to the entry of olfactory sensory axons into the olfactory bulb}

In addition to mistargeting of protoglomeruli by the axons of OR111-7 transgeneexpressing neurons in DCC and netrin $1 \mathrm{~b}$ knockdown larvae, we also observed errors in transgene-labeled axon trajectories between the olfactory epithelium and the bulb. Normally, olfactory axons exit the epithelium together in a tight bundle and traverse the short distance between the epithelium and the bulb by the most direct route. In contrast, in a majority of DCC MO1or DCC MO2-treated larvae, the axons of at least some OR111-7 transgene-expressing neurons project posteriorly and away from the olfactory bulb (Fig. $7 B$; see Fig. 9A, $B$, posterior category). Even in these animals, however, the majority of labeled sensory axons enter the bulb normally where they make the targeting errors described above. The same posterior misprojections between the olfactory epithelium and the bulb are observed at a much lower but still statistically significant rate in DCC mutant fish (Fig. 7C; see Fig. 9C, posterior category). Additional nonprotoglomerular misprojections are detected in DCC knockdown fish including small numbers of axons extending along the lateral-most margin of the bulb (Figs. $4 N, 7 E, F, H$; see Fig. 9A-C, lateral category). Notably, the protoglomerular and nonprotoglomerular errors quantified in Figures 6 and 9 are spread out throughout the whole population of analyzed olfactory bulbs. There is no apparent correlation between separate phenotypes, suggesting that they do not cluster together in any pattern that we could detect. Overall, the axons of a larger number of OR111-7 transgene-expressing neurons end in a variety of locations out- 


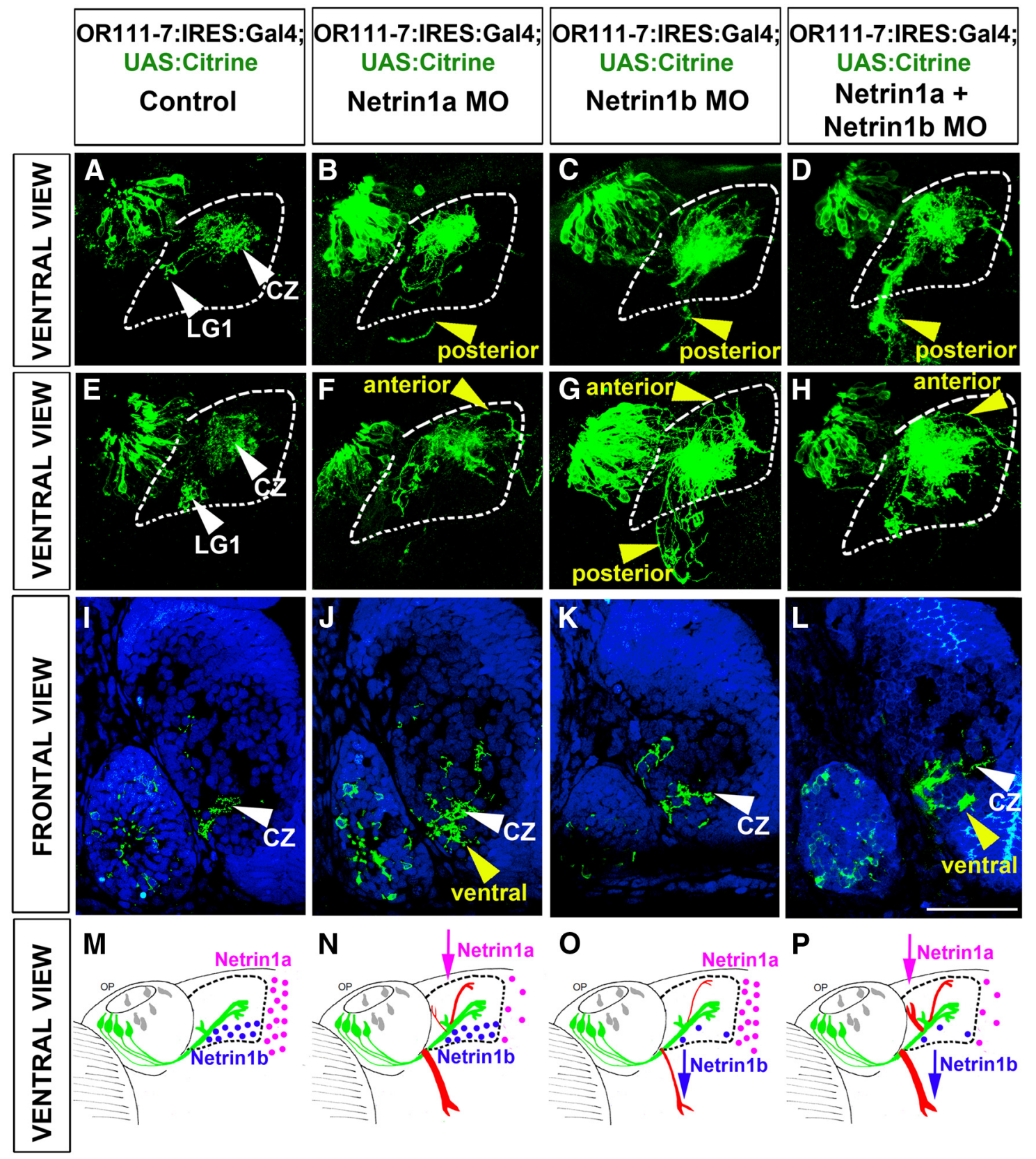

Figure 8. Netrin1a and netrin $1 \mathrm{~b}$ contribute to the proper pathfinding of OR111-7-transgenic axons into and within the olfactory bulb. $\boldsymbol{A}-\boldsymbol{H}$, Maximum intensity projections of serial confocal optical sections of 3-d-old larvae (ventral view). Anterior is to the top and the midline is to the right. The olfactory bulb is outlined with dashed lines. $\boldsymbol{M - P}$, Schematics showing control OR111-7:IRES:Gal4; UAS:Citrine projections (green) and mistargeted axons (red) observed upon inhibiting netrin1a, netrin1b or netrin1a and netrin1b together. The thickness of the red lines corresponds roughly to the penetrance of the indicated phenotypes. Netrin1a mRNA (pink dots) is expressed at the telencephalic midline and netrin $1 \mathrm{~b}$ (blue dots) is expressed in the ventral bulb. $A$, E, M, 0R111-7:IRES:Gal4; UAS:Citrine axons (green) project to the central zone (CZ, white arrowhead) and LG1. B-D, $\boldsymbol{N}-\boldsymbol{P}$, Some 0R111-7-transgenic axons misproject posteriorly (posterior, yellow arrowheads) rather than entering the olfactory bulb when Netrin1a, Netrin 1b, or both Netrin1a and Netrin1b levels are reduced. $\boldsymbol{F}-\boldsymbol{H}, \mathbf{N}-\boldsymbol{P}, \mathbf{0 R} 111-7$ :IRES:Gal4; UAS:(itrine axons also inappropriately stray anteriorly (anterior, yellow arrowheads) in netrin $1 \mathrm{a}$, netrin $1 \mathrm{~b}$, or netrin 1 and netrin $1 \mathrm{~b}$ double morphants. $I-L$, Single confocal optical sections of 3-d-old zebrafish larvae (frontal view). Dorsal is toward the top of the image. Propidium iodide (blue) labels olfactory bulb cells and allows identification of distinct protoglomeruli.I, OR111-7 transgene-expressing axons are observed in the central zone (CZ, white arrowhead) in uninjected Control larvae. J, L, Reducing Netrin1a levels or Netrin1a and Netrin1b levels together causes ventral mistargeting of OR111-7 transgeneexpressing axons (ventral, yellow arrowhead, schematized in Fig. $5 \boldsymbol{R}, \boldsymbol{T}$ ). Scale bar (in $\boldsymbol{L}$ ): $\boldsymbol{A}-\boldsymbol{L}, 50 \mu \mathrm{m}$.

side protoglomeruli in DCC knockdown as compared with normal larvae.

Very similar results are obtained in larvae injected with netrinla or netrin $1 \mathrm{~b}$ morpholinos, or both morpholinos together. Frequently the axons of OR111-7 transgene-expressing neurons were observed to turn posteriorly away from the bulb after exiting the olfactory epithelium (Figs. $8 A-D, M-P, 9 D-F$, posterior category). While the penetrance of posterior misprojec- tions is greater in netrinla morphants (59\%, Fig. 9D) compared with netrin $1 \mathrm{~b}$ morphants (30\%, Fig. $9 E$ ), the severity of posterior misprojections is greater in netrin $1 \mathrm{~b}$ morphants and the phenotype ranges from that shown in Figure $8 C$ to the one in $8 G$. Netrin la or Netrin $1 b$ knockdown is also observed to induce ectopic anterior trajectories of transgene-labeled axons (Figs. $8 E-$ $G, M-O, 9 D, E$, anterior category). Treatment of embryos with netrin $1 \mathrm{a}$ or with both netrin $1 \mathrm{a}$ and netrin $1 \mathrm{~b}$ morpholinos to- 

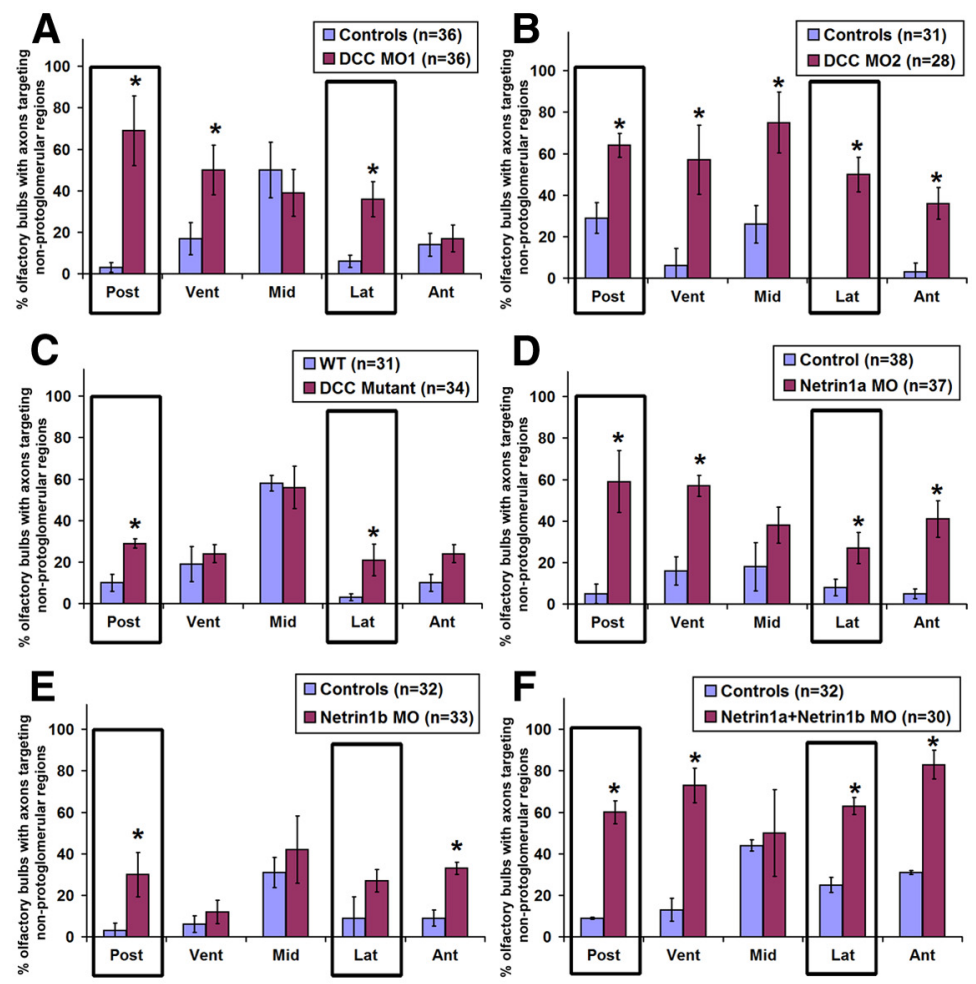

\section{G}

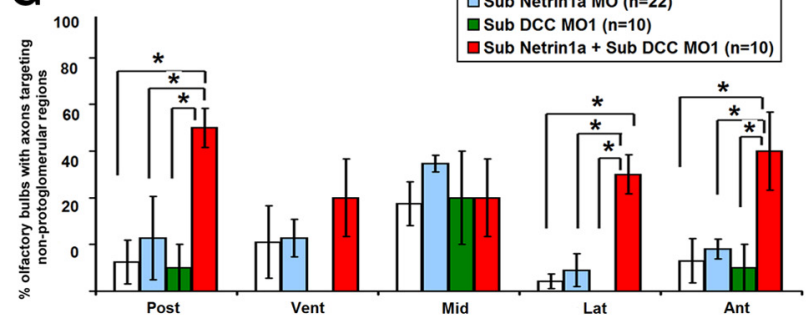

\section{$\square$ Controls ( $\mathrm{n}=24)$}

$\square$ Sub Netrin1a MO $(n=22)$ $\square$ Sub DCC MO1 $(\mathrm{n}=10)$

Figure 9. Quantification of non-protoglomerular targeting errors observed upon knocking down DCC, Netrin1a, Netrin1b, or both Netrins together. $A-G$, The percentages of larval olfactory bulbs in which OR111-7 transgene-expressing axons display the specified targeting errors are quantified. Error bars show the SEM. A-C, DCC M01 morphants, DCC M02 morphants, or DCC mutants (purple bars) are compared with control siblings (blue bars). $\boldsymbol{D}-\boldsymbol{F}$, Embryos injected with netrin1a $M 0$, netrin $1 \mathrm{~b} M 0$, or netrin1a and netrin $1 b$ MOs together (purple bars) are compared with uninjected control siblings (blue bars). G, The axonal projections of uninjected controls (white bars), subthreshold netrin $1 \mathrm{~b}$ morphants (blue bars), and subthreshold DCC morphants (green bars) are compared with morphants injected with subthreshold doses of both netrin $1 \mathrm{~b}$ and $D C C$ morpholinos together (red bars). $\boldsymbol{A}-\boldsymbol{F}$, The proportion of larvae with posterior misprojections (boxed) increases in DCC M01, DCC M02, netrin1a, netrin 1b, and netrin1a + netrin1b morphants, as well as in DCC mutant larvae. $\boldsymbol{A}-\boldsymbol{C}, \boldsymbol{D}, \boldsymbol{F}$, Reducing the levels of DCC, netrin1a or netrin1a together with netrin $1 b$ increases the number of larvae in which $0 R 111-7$ transgene-expressing axons make lateral pathfinding errors. $A, B, D, F$, DCC M01, DCC M02, netrin1a, and netrin1a + netrin1b morphants display increased ventral misprojections. $\boldsymbol{B}, \boldsymbol{D}-\boldsymbol{F}$, Larvae injected with DCC M02, netrin1a, netrin 1b, or netrin1a and netrin 1b morpholinos together display increased anterior misprojections. G, Embryos injected with subthreshold doses of netrin $1 \mathrm{a}$ and $\mathrm{DCC}$ morpholinos together display increased posterior, lateral, and anterior misprojections; but embryos injected with subthreshold doses of either netrin1a or DCC morpholino alone do not exhibit these guidance errors at more than control levels. Fisher's exact test was used to test for statistical significance, $p<0.05$. Post, Posterior; Vent, ventral; Mid, midline; Lat, lateral; Ant, anterior. These individual categories were previously grouped together as Other in Figure 6.

gether induce ventral misprojections of transgene-labeled axons (Figs. $5 Q, R, T, 8 I, J, L, 9 D, F$, ventral category). The netrinla and netrin $1 \mathrm{~b}$ morpholinos together induce an even higher rate of ectopic ventral, lateral, and anterior misprojections than either morpholino alone (Figs. 8D, $H, L, P, 9 D-F$ ). The parallel findings that both DCC and netrin knockdown induce similar misguidance effects supports a role for Netrin/DCC signaling in the axonal pathfinding of OR111-7 transgene-expressing sensory neurons. To examine the genetic interactions between DCC and the netrins in greater detail, we injected the individual morpho-

linos at subthreshold doses. Subthreshold knockdown of either netrin la or DCC individually does not induce posterior, lateral, or anterior misprojections (Fig. 9G). However, embryos injected with subthreshold doses of both netrin1a and DCC morpholinos display increased posterior, lateral, and anterior targeting errors. Similarly, subthreshold knockdown of netrin $1 \mathrm{~b}$ or DCC does not cause posterior misprojections, but embryos in which both netrin $1 \mathrm{~b}$ and DCC are reduced at subthreshold levels exhibit increased posterior misprojections (data not shown). These data show that netrins acting via the DCC receptor guide axons into and within the larval zebrafish olfactory bulb.

\section{Expression of DCC in}

\section{OR111-7-expressing neurons}

corrects mistargeting induced by

\section{DCC knockdown}

DCC is expressed in the olfactory epithelium, including most of the OR111-7 transgene-expressing subpopulation, and also in the olfactory bulb. In principle, netrin or DCC knockdown could affect sensory axon pathfinding either through a direct action on sensory axons themselves, or indirectly by influencing the development of netrin-sensitive cells within the bulb. To determine whether the axonal pathfinding defects we observed upon DCC knockdown were cell-autonomous to the OR111-7 transgene-expressing sensory neurons, we sought to increase DCC levels within this subpopulation in DCC morphants using a UAS: DCC-transgenic line (Lim et al., 2011). The UAS-driven DCC gene was modified so that it cannot be knocked down by the translation blocking DCC morpholino that we used in this study (See Materials and Methods). The UAS:DCC-transgenic construct contains a cardiac myosin light chain2 ( $\mathrm{cmlc2}$ ) promoter driving expression of mCherry. Thus, the presence of the UAS:DCC transgene was detected by selective red fluorescence in cardiac cells. OR111-7:IRES:Gal4;UAS:Citrine double transgenics were crossed to the UAS:DCC transgenic and injected with DCC MO1.

This generated two sets of larvae with fluorescently labeled olfactory sensory neurons: a set that overexpressed DCC in OR111-7 transgene- and Citrine-expressing neurons and a set that did not express DCC. Within each set, larvae were further subdivided into those injected with DCC morpholino at the single-cell stage or those that were not. Altogether there were a total of four sets of larvae: uninjected or morpholino-injected larvae that either overexpressed just Citrine or DCC and Citrine together. Expression of Citrine or DCC was confined exclusively to OR111-7 transgene-expressing neurons. We compared the axonal trajectories of uninjected fish that did 


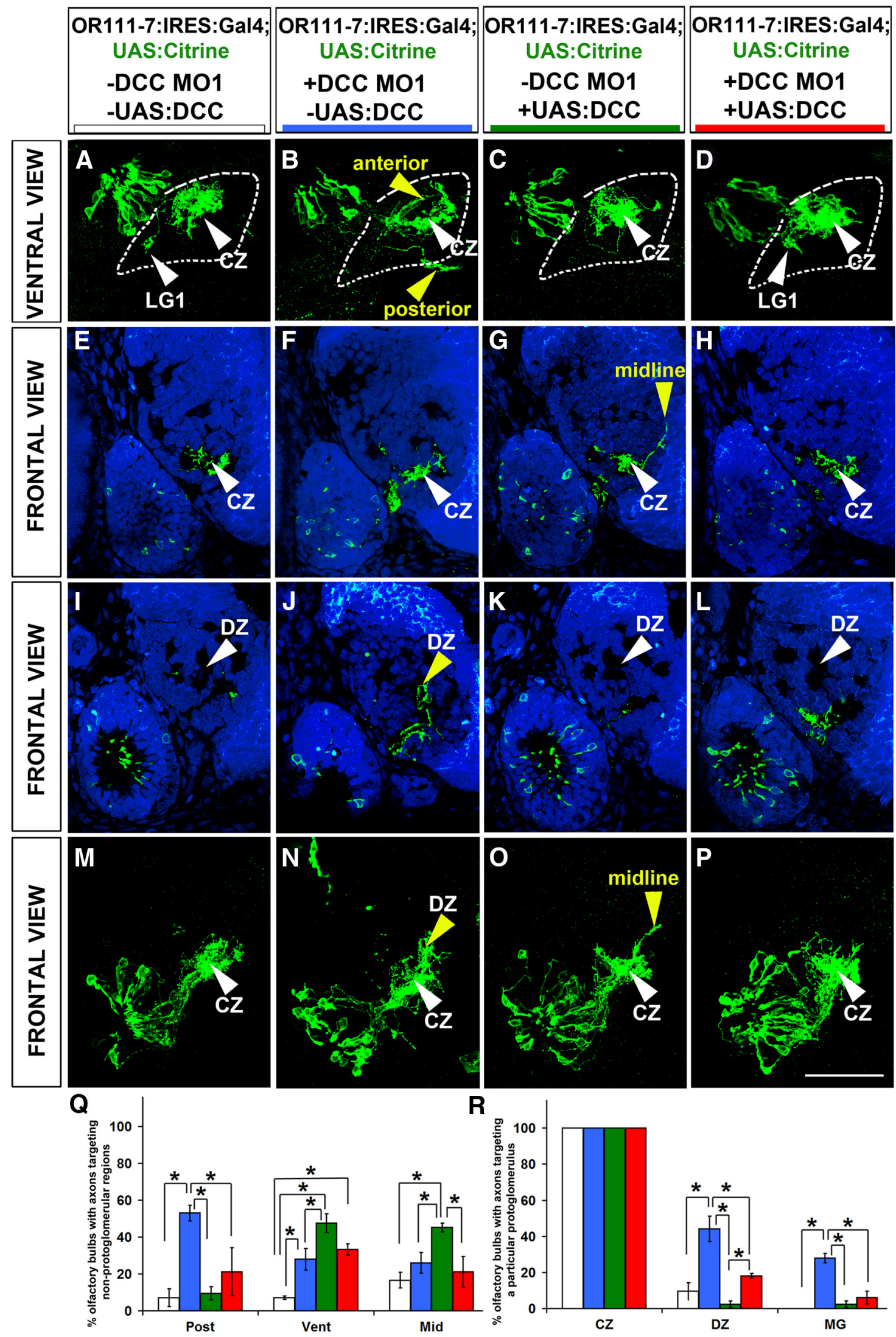

Figure 10. Expression of DCC in OR111-7-expressing neurons corrects mistargeting induced by $D C C$ knockdown. $A-D$, Maximum intensity projections of serial confocal optical sections of 3-d-old larvae (ventral view). $\boldsymbol{E}-\mathbf{L}$, Single confocal optical sections through 3-d-old OR111-7 transgene-expressing larvae (frontal view). Propidium iodide (blue) labels olfactory bulb cells and allows identification of distinct protoglomeruli. $\boldsymbol{M}-\boldsymbol{P}$, Maximum intensity projections of serial confocal optical sections of the same larvae shown immediately above. $\boldsymbol{Q}, \boldsymbol{R}$, The percentages of larval olfactory bulbs with the specified axonal misprojections are shown. Bars are color coded based on the larval groups indicated at the top of the figure. $A, E, I, M$, White bars in $\mathbf{Q}, \boldsymbol{R}$, Three-day-old control uninjected OR111-7:IRES:Gal4; UAS:Citrine larvae without the UAS:DCC transgene. $\boldsymbol{B}, \boldsymbol{F}, \boldsymbol{J}, \mathbf{N}$, Blue bars in $\mathbf{Q}, \boldsymbol{R}$, Three-day-old 0R111-7:IRES:Gal4; UAS:Citrine larvae without (Figure legend continues.) 
not express the DCC transgene (Fig. 10A,E,I,M, white bars in $Q, R)$, fish injected with a DCC morpholino that did not express the DCC transgene (Fig. $10 B, F, J, N$, blue bars in $Q, R$ ), uninjected fish that expressed the DCC transgene (Fig. 10C, G, K, O, green bars in $Q, R)$, and fish injected with a DCC morpholino that expressed the $D C C$ transgene (Fig. $10 D, H, L, P$, red bars in $Q, R$ ). As expected, knocking down DCC levels induced posterior misprojections (Fig. 10A, $B$, blue Post bar in $Q$ ), and also dorsal misprojections to the DZ and MG protoglomeruli (Fig. $10 I, J, M, N$, blue $\mathrm{DZ}$ and MG bars in $R$ ). These morpholino-induced errors were partially corrected to a significant degree in larvae that overexpressed DCC only in the OR111-7 transgene-expressing neurons (Fig. $10 D, H, L, P$, red Post bar in $Q$, red DZ and MG bars in $R$ ). Overexpression of DCC in the OR111-7-expressing neurons of non-morpholino-treated fish had no significant effect on these particular errors. But interestingly, overexpression of DCC in OR111-7-expressing neurons did increase the chance that their axons would misproject ventrally and to the midline (Fig. 10G,O, green Vent and Mid bars in Q). These results are consistent with a requirement for DCC in the subset of olfactory sensory neurons that express the OR111-7 transgene for correct targeting of the central zone protoglomerulus. They also suggest that the overexpression of DCC in the same cells can drive their axons closer to the midline and ventral margin of the olfactory bulb where netrins are expressed, further supporting the idea that Netrins act as attractants for these axons.

\section{Discussion}

Vertebrate olfactory sensory neurons choose to express a single odorant receptor from a large genomic repertoire. This choice of odorant receptor determines the odorant specificity of the neuron. The axons of neurons that express a particular odorant receptor reproducibly target selected glomeruli in the olfactory bulb. Odorants evoke a characteristic activation pattern of one or more specific glomeruli, revealing an underlying coding mechanism for odorants in the primary olfactory circuit (Friedrich and Korsching, 1997, 1998; Rubin and Katz., 1999; Belluscio and Katz., 2001). The formation of an accurate map of olfactory sensory axons into reproducibly located glomeruli in the bulb requires that sensory neurons expressing particular receptors be guided to specific locations in the bulb. However, they do not converge into glomeruli immediately, but first form protoglo-

\footnotetext{
$\leftarrow$

(Figure legend continued.) UAS:DCC were injected with DCC M01. C, G, $\boldsymbol{K}, \mathbf{0}$, Green bars in $\mathbf{Q}, \boldsymbol{R}$, Three-day-old uninjected OR111-7:IRES:Gal4; UAS:Citrine larvae that also carry the UAS:DCC transgene. $\boldsymbol{D}, \boldsymbol{H}, \boldsymbol{L}, \boldsymbol{P}$, Red bars in $\mathbf{Q}, \boldsymbol{R}$, Three-day-old 0R111-7:IRES:Gal4; UAS: Citrine larvae carrying UAS:DCC were injected with DCC M01 (Rescue condition). $\boldsymbol{B}$, Blue Post bar in $\mathbf{Q}$, Reducing DCC levels causes posterior misprojections. $\boldsymbol{A}, \boldsymbol{C}$, White and green Post bars in $\mathbf{Q}$, Posterior targeting errors are not observed in the absence of DCC MO. OR111-7-transgenic axons (green) are present in the central zone (CZ, white arrowhead) and in the lateral glomerulus 1 (LG1, white arrowhead). D, Red Post bar in $\mathbf{Q}$, Posterior targeting errors in DCC morphants are reduced in OR111-7 axons also expressing the UAS:DCC transgene. J, $\boldsymbol{N}$, Blue DZ bar in $\boldsymbol{R}$, Reducing DCC levels causes 0R111-7-transgenic neurons to inappropriately enter the $D Z$ (yellow arrowhead). $\boldsymbol{I}, \boldsymbol{M}, \boldsymbol{K}, \boldsymbol{O}$, White and green $D Z$ bars in $R, 0 R 111-7$ transgene-expressing axons do not enter $D Z$ in the absence of $D C C$ morpholino regardless of whether the UAS:DCC transgene is present. $\boldsymbol{L}, \boldsymbol{P}$, Red DZ bar in $\boldsymbol{R}$, Axonal mistargeting into $D Z$ is reduced in DCC morphants that contain the morpholinoresistant UAS:DCC transgene. Green Vent and Mid bars in $\mathbf{Q}$, Increased ventral and midline misprojections are observed in 0R111-7-transgenic larvae that express the UAS:DCC transgene. Fisher's exact test was used to test for statistical significance, $p<0.05$. Scale bar (in $\boldsymbol{P}): \boldsymbol{A}-\boldsymbol{P}, 50 \mu \mathrm{m}$. Post, Posterior; Vent, ventral; Mid, midline; CZ, central zone.
}

merular condensations from which individual glomeruli segregate over time.

Protoglomeruli have been observed not only in zebrafish ( $\mathrm{Li}$ et al., 2005), but also in insects and rodents (Oland et al., 1990; Treloar et al., 1999; Conzelmann et al., 2001). There are 12 distinct, identifiable protoglomeruli in the 3-d-old zebrafish olfactory bulb (Dynes and Ngai, 1998). They are innervated by at least two classes of olfactory sensory neurons in the olfactory epithelium: ciliated neurons expressing classical ORs along with OMP, and microvillous neurons expressing V2R-type vomeronasal receptors along with TRPC2. Selective expression of fluorophores directed by OMP or TRPC2 promoters in transgenic animals showed that OMP and TRPC2 axons target distinct protoglomeruli in the bulb (Sato et al., 2005). Our work extends these findings by defining the particular protoglomeruli innervated by these two general classes of sensory axons. The OMP-expressing class of sensory axons terminate in the central zone, dorsal zone, lateral protoglomerulus 3, and a very few axons in MG1-4 protoglomeruli. TRPC2-expressing axons terminate in the olfactory plexus, lateral protoglomeruli 1, 2 and 4; and the ventroposterior protoglomerulus. Our work further suggests that there is at least one additional class of sensory neurons that express neither OMP nor TRPC2. Since the medial protoglomeruli are only very sparsely innervated by either of these two classes, it is attractive to hypothesize that they are innervated by olfactory sensory neurons expressing the recently discovered TAAR and/or V1R class of receptors (Saraiva and Korsching, 2007; Hussain et al., 2009).

Our results reveal that the Netrin receptor DCC is required cell autonomously within a small subpopulation of sensory neurons for their axons to correctly target the central zone protoglomerulus. Reducing the expression or activity of DCC, Netrin 1a or Netrin $1 b$ interferes with the ability of axons from the OR111-7 transgene-expressing subpopulation of neurons to enter the olfactory bulb and to converge upon the central zone protoglomerulus. These findings are consistent with an attractive function for Netrin la expressed at the midline and Netrin $1 \mathrm{~b}$ expressed ventrally where sensory axons enter the bulb. Reducing DCC or Netrinlb levels induces the axons of transgeneexpressing neurons that enter the bulb to mistarget dorsal protoglomeruli. These data suggest that Netrin $1 b$ expressed in the ventral portion of the bulb helps draw these axons into the central zone protoglomerulus and prevents them from wandering into more dorsal protoglomeruli. Altogether, we propose that a combination of midline Netrinla and ventral Netrin $1 b$ signaling helps to guide a specific population of axons into the bulb and to terminate within the central zone protoglomerulus.

Netrin/DCC signaling has been shown to mediate attractive effects in a wide variety of axons in invertebrates (Hedgecock et al., 1990; Kolodziej et al., 1996; Mitchell et al., 1996), in vertebrate central commissures (Kennedy et al., 1994; Serafini et al., 1996; Fazeli et al., 1997), and in noncommissural projections in vertebrates including corticofugal and thalamocortical axons (Métin et al., 1997; Braisted et al., 2000). DCC has been detected on rat olfactory axons that are extending toward the telencephalon and Netrin 1 is present along their trajectory, suggesting that Netrin/ DCC signaling could potentially play a role in early olfactory map formation in rodents (Astic et al., 2002). Netrins can bind and act through other alternative receptors. Unc-5, in combination with DCC or on its own, mediates a repulsive response to netrins (Hong et al., 1999). In principle, a repellent reaction to netrin expressed at the midline in zebrafish could help guide axons that terminate in more lateral protoglomeruli. There are three known Unc5 genes in zebrafish: Unc5a, Unc5b, and Unc5c. We did not 
detect any of their mRNAs by in situ hybridization in the developing olfactory epithelium. Unc5a and Unc 5 c mRNA were, however, detected in the developing olfactory bulb along with DCC mRNA. It is therefore unlikely that Unc5 mediated repellent responses to netrins guide olfactory sensory axons within the bulb, but it remains a possibility that repellent netrin signaling contributes to the protoglomerular targeting of mitral cell dendrites. Neogenin is a DCC-like receptor that also binds Netrin 1, albeit an order of magnitude less potently than its other ligand, repulsive guidance molecule RGM (Rajagopalan et al., 2004). We found that neogenin 1 mRNA is detected in 27 hpf OMP- and OR1117-expressing neurons by in situ hybridization. It is possible that Neogenin 1 acts in concert with DCC to attract olfactory sensory axons to Netrin1-expressing areas. Neogenin 1 morphants have defects in neural tube formation and neuronal differentiation, thus impeding the analysis of its role in olfactory axonal guidance (Mawdsley et al., 2004). The requirement for Neogenin 1 in axonal targeting could be tested in future studies by expressing a dominant-negative Neogenin 1 receptor in subpopulations of olfactory sensory neurons.

Several other traditional guidance cues have been implicated in organizing the mouse glomerular map. The repulsive cues Slit 1 and Slit3 are present in the ventral olfactory bulb in mice while OSNs in the epithelium express the Slit receptor, Robo2. In both Robo 2 mutant mice and slit 1 mutant mice, a subset of OSNs that normally targets the dorsal region of the bulb misprojects ventrally and forms ectopic glomeruli (Cho et al., 2007). Some olfactory glomeruli in Robo2 mutant zebrafish are either absent or ectopically located. This phenotype has been ascribed to global protoglomerular disruption in Robo2 mutant larvae (Miyasaka et al., 2005). In developing Xenopus spinal axons, the activated Robo receptor has been reported to bind DCC and silence the attractive effect of $\mathrm{Ne}$ trin1 (Stein and Tessier-Lavigne, 2001). Inhibition of Slit/ Robo signaling in chick and mouse motor neurons induces axonal grown toward Netrin 1 (Bai et al., 2011). We wondered whether the guidance of OR111-7 transgene-expressing axons depends upon Robo receptor signaling, either directly or through modulation of Netrin/DCC signaling. We have examined the trajectories of OR111-7 transgene-expressing axons in zebrafish embryos with a null mutation in robo2 and find no consistent errors (data not shown). However, our results to date cannot exclude the possibility that the targeting of other olfactory sensory axons is affected by the loss of Robo2mediated signaling.

The repulsive ligand Sema3F is expressed in the dorsal bulb of mice and repels axons that express its receptor, Neuropilin2, thus confining these axons to the ventral bulb. Knocking down either Sema3F or Neuropilin2 in olfactory sensory neurons mistargets ventrally projecting axons to the dorsal olfactory bulb (Takeuchi et al., 2010). Sema3a is expressed in mice by ensheathing cells in the nerve layer of the ventral olfactory bulb while some olfactory sensory neurons express its receptor, Neuropilin1a. Neuropilin1a-positive axons extend to the lateral region of the anterior olfactory bulb and medial region of the posterior bulb, avoiding Sema3A-expressing regions. In Sema3A deficient mice, many Neuropilin1a-positive glomeruli are ectopically positioned in the anteromedial and ventral bulb (Schwarting et al., 2000, 2004; Taniguchi et al., 2003). The repulsive guidance mediated by Neuropilin-1 receptor signaling in olfactory sensory axons seems to be conserved to some degree across vertebrate species. Chick olfactory sensory neurons expressing a dominant-negative Neuropilin 1 enter the brain prematurely and overshoot Semaphorin-expressing regions in the bulb to project into the forebrain (Renzi et al., 2000). Finally, deleting ephrinA3 and ephrinA5 together in mice shifts glomeruli of SR1 or P2 odorant receptor-expressing olfactory sensory neurons posteriorly, while overexpression of EphrinA5 specifically in P2 neurons shifts its glomerulus anteriorly (Cutforth et al., 2003). These studies have uncovered a crucial role for axonal repellents in olfactory map formation. Our study, however, is the first to demonstrate the requirement for attractive cues, specifically netrins, in proper targeting of olfactory sensory axons.

Although studies in the mouse have identified several cues that contribute to olfactory axon guidance, the complexity and inaccessibility of its developing olfactory system has limited most observations to postnatal animals in which the glomeruli are almost fully differentiated. Thus the developmental contribution of these signaling molecules to initial targeting is not always clear, and the processes that drive the segregation of axons into glomeruli may confound the analysis of some axonal guidance mutants. By comparison, the zebrafish is an excellent vertebrate system in which to study the initial targeting of olfactory sensory axons in the olfactory bulb. The system is relatively simple, with $\sim 150$ ORs compared with $>1000$ in mice (Alioto and Ngai, 2005). Development is rapid, with initial axon targeting occurring within the first $3 \mathrm{~d}$ of fertilization (Whitlock and Westerfield, 1998; Wilson et al., 1990; Sato et al., 2005). The embryo is small and transparent, making it possible to observe sensory axon pathfinding even in a living animal (Dynes and Ngai, 1998). Most importantly, sensory axons entering the bulb first terminate in distinct individually identifiable neuropilar protoglomeruli.

Our results highlight the advantages of a relatively simple vertebrate olfactory system for studying the initial targeting of olfactory sensory axons to defined protoglomeruli in the olfactory bulb. In this study, we identified for the first time in any model system, an important role for Netrin/DCC signaling in the correct initial targeting of axons from the olfactory epithelium to the bulb and within the bulb itself. To deduce the overall molecular logic underlying protoglomerular targeting, it will be necessary to generate additional transgenic lines that label axons that target protoglomeruli other than the central zone. Using the OR111-7-transgenic line and additional lines as they become available, it will be possible to assess the specific contributions of almost any candidate guidance cue to the mapping of specific olfactory sensory neurons to their targets in the olfactory bulb.

\section{References}

Alioto TS, Ngai J (2005) The odorant receptor repertoire of teleost fish. BMC Genomics 6:173.

Astic L, Pellier-Monnin V, Saucier D, Charrier C, Mehlen P (2002) Expression of netrin-1 and netrin-1 receptor, DCC, in the rat olfactory nerve pathway during development and axonal regeneration. Neuroscience 109:643-656

Bai G, Chivatakarn O, Bonanomi D, Lettieri K, Franco L, Xia C, Stein E, Ma L, Lewcock JW, Pfaff SL (2011) Presenilin-dependent receptor processing is required for axon guidance. Cell 144:106-118.

Balciunas D, Wangensteen KJ, Wilber A, Bell J, Geurts A, Sivasubbu S, Wang X, Hackett PB, Largaespada DA, McIvor RS, Ekker SC (2006) Harnessing a high cargo-capacity transposon for genetic applications in vertebrates. PLoS Genet 2:e169.

Barth AL, Dugas JC, Ngai J (1997) Noncoordinate expression of odorant receptor genes tightly linked in the zebrafish genome. Neuron 19:359-369.

Belluscio L, Katz LC (2001) Symmetry, stereotypy, and topography of odorant representations in mouse olfactory bulbs. J Neurosci 21:2113-2122.

Bonkowsky JL, Wang X, Fujimoto E, Lee JE, Chien CB, Dorsky RI (2008) 
Domain-specific regulation of foxP2 CNS expression by lef1. BMC Dev Biol 8:103.

Bozza T, Vassalli A, Fuss S, Zhang JJ, Weiland B, Pacifico R, Feinstein P, Mombaerts P (2009) Mapping of class I and class II odorant receptors to glomerular domains by two distinct types of olfactory sensory neurons in the mouse. Neuron 61:220-233.

Braisted JE, Catalano SM, Stimac R, Kennedy TE, Tessier-Lavigne M, Shatz CJ, O'Leary DD (2000) Netrin-1 promotes thalamic axon growth and is required for proper development of the thalamocortical projection. J Neurosci 20:5792-5801.

Brend T, Holley SA (2009) Zebrafish whole mount high-resolution double fluorescent in situ hybridization. J Vis Exp 25:pii: 1229. doi:10.3791/1229.

Celik A, Fuss SH, Korsching SI (2002) Selective targeting of zebrafish olfactory receptor neurons by the endogenous OMP promoter. Eur J Neurosci 15:798-806.

Chalasani SH, Sabol A, Xu H, Gyda MA, Rasband K, Granato M, Chien CB, Raper JA (2007) Stromal cell-derived factor-1 antagonizes slit/robo signaling in vivo. J Neurosci 27:973-980.

Cho JH, Lépine M, Andrews W, Parnavelas J, Cloutier JF (2007) Requirement for slit-1 and robo-2 in zonal segregation of olfactory sensory neuron axons in the main olfactory bulb. J Neurosci 27:9094-9104.

Conzelmann S, Malun D, Breer H, Strotmann J (2001) Brain targeting and glomerulus formation of two olfactory neuron populations expressing related receptor types. Eur J Neurosci 14:1623-1632.

Cutforth T, Moring L, Mendelsohn M, Nemes A, Shah NM, Kim MM, Frisén J, Axel R (2003) Axonal ephrin-as and odorant receptors: Coordinate determination of the olfactory sensory map. Cell 114:311-322.

Dynes JL, Ngai J (1998) Pathfinding of olfactory neuron axons to stereotyped glomerular targets revealed by dynamic imaging in living zebrafish embryos. Neuron 20:1081-1091.

Fazeli A, Dickinson SL, Hermiston ML, Tighe RV, Steen RG, Small CG, Stoeckli ET, Keino-Masu K, Masu M, Rayburn H, Simons J, Bronson RT, Gordon JI, Tessier-Lavigne M, Weinberg RA (1997) Phenotype of mice lacking functional deleted in colorectal cancer (dcc) gene. Nature 386:796-804.

Fisher S, Grice EA, Vinton RM, Bessling SL, Urasaki A, Kawakami K, McCallion AS (2006) Evaluating the biological relevance of putative enhancers using Tol2 transposon-mediated transgenesis in zebrafish. Nat Protoc $1: 1297-1305$.

Fricke C, Chien CB (2005) Cloning of full-length zebrafish dcc and expression analysis during embryonic and early larval development. Dev Dyn 234:732-739.

Friedrich RW, Korsching SI (1997) Combinatorial and chemotopic odorant coding in the zebrafish olfactory bulb visualized by optical imaging. Neuron 18:737-752.

Friedrich RW, Korsching SI (1998) Chemotopic, combinatorial, and noncombinatorial odorant representations in the olfactory bulb revealed using a voltage-sensitive axon tracer. J Neurosci 18:9977-9988.

Granato M, van Eeden FJ, Schach U, Trowe T, Brand M, Furutani-Seiki M, Haffter P, Hammerschmidt M, Heisenberg CP, Jiang YJ, Kane DA, Kelsh RN, Mullins MC, Odenthal J, Nüsslein-Volhard C (1996) Genes controlling and mediating locomotion behavior of the zebrafish embryo and larva. Development 123:399-413.

Hamdani el H, Døving KB (2007) The functional organization of the fish olfactory system. Prog Neurobiol 82:80-86.

Hedgecock EM, Culotti JG, Hall DH (1990) The unc-5, unc-6, and unc-40 genes guide circumferential migrations of pioneer axons and mesodermal cells on the epidermis in C. elegans. Neuron 4:61-85.

Hong K, Hinck L, Nishiyama M, Poo MM, Tessier-Lavigne M, Stein E (1999) A ligand-gated association between cytoplasmic domains of UNC5 and DCC family receptors converts netrin-induced growth cone attraction to repulsion. Cell 97:927-941.

Hussain A, Saraiva LR, Korsching SI (2009) Positive darwinian selection and the birth of an olfactory receptor clade in teleosts. Proc Natl Acad Sci U S A 106:4313-4318.

Imai T, Suzuki M, Sakano H (2006) Odorant receptor-derived cAMP signals direct axonal targeting. Science 314:657-661.

Ishii N, Wadsworth WG, Stern BD, Culotti JG, Hedgecock EM (1992) UNC-6, a laminin-related protein, guides cell and pioneer axon migrations in C. elegans. Neuron 9:873-881.

Kennedy TE, Serafini T, de la Torre JR, Tessier-Lavigne M (1994) Netrins are diffusible chemotropic factors for commissural axons in the embryonic spinal cord. Cell 78:425-435.

Kolodziej PA, Timpe LC, Mitchell KJ, Fried SR, Goodman CS, Jan LY, Jan YN (1996) Frazzled encodes a Drosophila member of the DCC immunoglobulin subfamily and is required for CNS and motor axon guidance. Cell 87:197-204.

Kwan KM, Fujimoto E, Grabher C, Mangum BD, Hardy ME, Campbell DS, Parant JM, Yost HJ, Kanki JP, Chien CB (2007) The Tol2kit: A multisite gateway-based construction kit for Tol2 transposon transgenesis constructs. Dev Dyn 236:3088-3099.

Li J, Mack JA, Souren M, Yaksi E, Higashijima S, Mione M, Fetcho JR, Friedrich RW (2005) Early development of functional spatial maps in the zebrafish olfactory bulb. J Neurosci 25:5784-5795.

Lim AH, Suli A, Yaniv K, Weinstein B, Li DY, Chien CB (2011) Motoneurons are essential for vascular pathfinding. Development 138:3847-3857.

Mawdsley DJ, Cooper HM, Hogan BM, Cody SH, Lieschke GJ, Heath JK (2004) The Netrin receptor Neogenin is required for neural tube formation and somitogenesis in zebrafish. Dev Biol 269:302-315.

Métin C, Deléglise D, Serafini T, Kennedy TE, Tessier-Lavigne M (1997) A role for netrin-1 in the guidance of cortical efferents. Development 124:5063-5074.

Mitchell KJ, Doyle JL, Serafini T, Kennedy TE, Tessier-Lavigne M, Goodman CS, Dickson BJ (1996) Genetic analysis of netrin genes in Drosophila: Netrins guide CNS commissural axons and peripheral motor axons. Neuron 17:203-215.

Miyasaka N, Sato Y, Yeo SY, Hutson LD, Chien CB, Okamoto H, Yoshihara Y (2005) Robo2 is required for establishment of a precise glomerular map in the zebrafish olfactory system. Development 132:1283-1293.

Mombaerts P, Wang F, Dulac C, Chao SK, Nemes A, Mendelsohn M, Edmondson J, Axel R (1996) Visualizing an olfactory sensory map. Cell $87: 675-686$.

Nishizumi H, Kumasaka K, Inoue N, Nakashima A, Sakano H (2007) Deletion of the core- $\mathrm{H}$ region in mice abolishes the expression of three proximal odorant receptor genes in cis. Proc Natl Acad Sci U S A 104:20067-20072.

Oland LA, Orr G, Tolbert LP (1990) Construction of a protoglomerular template by olfactory axons initiates the formation of olfactory glomeruli in the insect brain. J Neurosci 10:2096-2112.

Rajagopalan S, Deitinghoff L, Davis D, Conrad S, Skutella T, Chedotal A, Mueller BK, Strittmatter SM (2004) Neogenin mediates the action of repulsive guidance molecule. Nat Cell Biol 6:756-762.

Renzi MJ, Wexler TL, Raper JA (2000) Olfactory sensory axons expressing a dominant-negative semaphorin receptor enter the CNS early and overshoot their target. Neuron 28:437-447.

Robu ME, Larson JD, Nasevicius A, Beiraghi S, Brenner C, Farber SA, Ekker SC (2007) P53 activation by knockdown technologies. PLoS Genet 3:e78.

Rubin BD, Katz LC (1999) Optical imaging of odorant representations in the mammalian olfactory bulb. Neuron 23:499-511.

Sakano H (2010) Neural map formation in the mouse olfactory system. Neuron 67:530-542.

Saraiva LR, Korsching SI (2007) A novel olfactory receptor gene family in teleost fish. Genome Res 17:1448-1457.

Sato Y, Miyasaka N, Yoshihara Y (2005) Mutually exclusive glomerular innervation by two distinct types of olfactory sensory neurons revealed in transgenic zebrafish. J Neurosci 25:4889-4897.

Sato Y, Miyasaka N, Yoshihara Y (2007) Hierarchical regulation of odorant receptor gene choice and subsequent axonal projection of olfactory sensory neurons in zebrafish. J Neurosci 27:1606-1615.

Schwarting GA, Kostek C, Ahmad N, Dibble C, Pays L, Püschel AW (2000) Semaphorin $3 \mathrm{~A}$ is required for guidance of olfactory axons in mice. J Neurosci 20:7691-7697.

Schwarting GA, Raitcheva D, Crandall JE, Burkhardt C, Püschel AW (2004) Semaphorin 3A-mediated axon guidance regulates convergence and targeting of P2 odorant receptor axons. Eur J Neurosci 19:1800-1810.

Serafini T, Kennedy TE, Galko MJ, Mirzayan C, Jessell TM, Tessier-Lavigne M (1994) The netrins define a family of axon outgrowth-promoting proteins homologous to C. elegans UNC-6. Cell 78:409-424.

Serafini T, Colamarino SA, Leonardo ED, Wang H, Beddington R, Skarnes 
WC, Tessier-Lavigne M (1996) Netrin-1 is required for commissural axon guidance in the developing vertebrate nervous system. Cell 87:1001-1014.

Shykind BM (2005) Regulation of odorant receptors: One allele at a time. Hum Mol Genet 14 Spec No 1:R33-9.

Stein E, Tessier-Lavigne M (2001) Hierarchical organization of guidance receptors: silencing of netrin attraction by slit through a Robo/DCC receptor complex. Science 291:1928-1938.

Suli A, Mortimer N, Shepherd I, Chien CB (2006) Netrin/DCC signaling controls contralateral dendrites of octavolateralis efferent neurons. J Neurosci 26:13328-13337.

Takeuchi H, Inokuchi K, Aoki M, Suto F, Tsuboi A, Matsuda I, Suzuki M, Aiba A, Serizawa S, Yoshihara Y, Fujisawa H, Sakano H (2010) Sequential arrival and graded secretion of Sema3F by olfactory neuron axons specify map topography at the bulb. Cell 141:1056-1067.
Taniguchi M, Nagao H, Takahashi YK, Yamaguchi M, Mitsui S, Yagi T, Mori K, Shimizu T (2003) Distorted odor maps in the olfactory bulb of semaphorin 3A-deficient mice. J Neurosci 23:1390-1397.

Treloar HB, Purcell AL, Greer CA (1999) Glomerular formation in the developing rat olfactory bulb. J Comp Neurol 413:289-304.

Vassalli A, Rothman A, Feinstein P, Zapotocky M, Mombaerts P (2002) Minigenes impart odorant receptor-specific axon guidance in the olfactory bulb. Neuron 35:681-696.

Wang F, Nemes A, Mendelsohn M, Axel R (1998) Odorant receptors govern the formation of a precise topographic map. Cell 93:47-60.

Whitlock KE, Westerfield M (1998) A transient population of neurons pioneers the olfactory pathway in the zebrafish. J Neurosci 18:8919-8927.

Wilson SW, Ross LS, Parrett T, Easter SS Jr (1990) The development of a simple scaffold of axon tracts in the brain of the embryonic zebrafish, Brachydanio rerio. Development 108:121-145. 